\title{
TRACES OF INTERTWINERS FOR QUANTUM GROUPS AND DIFFERENCE EQUATIONS, I
}

\author{
Pavel Etingof and Alexander Varchenko \\ Department of Mathematics, Rm 2-165 \\ MIT, 77 Mass. Ave. \\ Cambridge, MA 02139, USA \\ e-mail: etingof@math.harvard.edu \\ and \\ Department of Mathematics \\ University of North Carolina \\ Chapel Hill, NC 27599, USA \\ e-mail: av@math.unc.edu
}

\section{INTRODUCTION}

This paper begins a series of papers whose goal is to establish a representationtheoretic interpretation of the quantum Knizhnik-Zamolodchikov-Bernard (qKZB) equations, and use this interpretation to study solutions of these equations. It was motivated by the recent work on the qKZB equations [F,FTV1-2,MV,FV2-5], and by the theory of "quantum conformal blocks" that began with the classical paper $[\mathrm{FR}]$.

0.1. The qKZB equations $[\mathrm{F}]$ are difference equations with respect to an unknown function $f\left(z_{1}, \ldots, z_{N}, \lambda, \tau, \mu, p\right)$ with values in $V_{1} \otimes \ldots \otimes V_{N} \otimes V_{N}^{*} \otimes \ldots \otimes V_{1}^{*}$, where $V_{i}$ are suitable finite dimensional representations of the quantum group $U_{q}(\mathfrak{g})$ ( $\mathfrak{g}$ is a simple Lie algebra), $z_{i}, p, \tau \in \mathbb{C}$, and $\lambda, \mu$ are weights for $\mathfrak{g}$.

The qKZB equations are a q-deformation of the Knizhnik-Zamolodchikov-Bernard (KZB) differential equations, and an elliptic analogue of the quantum KnizhnikZamolodchikov (qKZ) difference equations, which are, in turn, generalizations of the usual (trigonometric) Knizhnik-Zamolodchikov equations.

It is proved in [FTV2] (using an integral representation of solutions) that for $\mathfrak{g}=s l_{2}$ the monodromy of the qKZB equations is given by the dual qKZB equations, which are obtained from the qKZB equations by interchanging $(\lambda, \tau)$ with $(\mu, p)$. This fact generalizes the monodromy theorems for the KZB and $\mathrm{qKZ}$ equations: the monodromy of the KZB differential equations is the trigonometric degeneration of the qKZB equations (which involves dynamical R-matrices without spectral parameter) (see e.g. $[\mathrm{K}]$ ), and the monodromy of the $\mathrm{qKZ}$ equations is given by elliptic dynamical R-matrices (but there is no difference equation) ([TV1,TV2]; see also $[\mathrm{FR}])$. 
The self-duality of the qKZB equations leads one to expect that they should have symmetric solutions $u_{V_{1}, \ldots, V_{N}}(z, \lambda, \tau, \mu, p)$, i.e. such that $u_{V_{1}, \ldots, V_{N}}(z, \lambda, \tau, \mu, p)=$ $u_{V_{N}^{*}, \ldots, V_{1}^{*}}^{*}(z, \mu, p, \lambda, \tau)$, where $u^{*}$ is the dual of $u$ (considered as an endomorphism of $\left.V_{1} \otimes \ldots \otimes V_{N}\right)$. Such a solution $u\left(\right.$ for $\mathfrak{g}=s l_{2}$ ) was constructed in [FV2,FTV2], by an explicit integral formula. It is called the universal hypergeometric function. This function has many interesting properties, in particular the $S L(3, \mathbb{Z})$-symmetry [FV3-5], where the group $S L(3, \mathbb{Z})$ acts on the lattice $\mathbb{Z}^{3}$ generated by the periods $1, \tau, p$. A consequence of this symmetry is the qKZB-heat equation [FV2] for the function $u$, which is a q-deformation of the KZB-heat equation [Ber].

0.2. A central fact about the KZB and $q K Z$ equations (and one of the main reasons why they are interesting) is that they are satisfied by conformal blocks. More precisely, the KZB equations are satisfied by conformal blocks of the Wess-ZuminoWitten conformal field theory on an elliptic curve [Ber], and the qKZ equations are satisfied by quantum conformal blocks on the cylinder $[\mathrm{FR}]$. In representation theoretic terms, conformal blocks on an elliptic curve are traces of products of intertwining operators for affine Lie algebras (weighted by an element from the maximal torus) [Ber], and quantum conformal blocks on the cylinder are highest matrix elements of products of intertwining operators for quantum affine algebras[FR]. This representation theoretic interpretation of the KZB and $\mathrm{qKZ}$ equations is not only interesting by itself, but it also allows to prove nontrivial properties of solutions, e.g. monodromy theorems (see e.g. $[\mathrm{K}, \mathrm{FR}]$ ).

The goal of this series is to give a similar interpretation of the qKZB equations. In light of the above, the main idea is obvious: one should consider quantum conformal blocks on an elliptic curve, or, representation theoretically, traces of products of intertwining operators for quantum affine algebras, weighted by an element of the maximal torus. It is natural to expect that such traces satisfy a pair of dual qKZB equations. This is actually true, and we plan to give a proof of it in a subsequent part of the series. However, the details of the proof are relatively complicated, and we would like to start with a simpler ("trigonometric") limiting case, when $\tau, p \rightarrow \infty$. This limiting case is the main subject of this paper.

0.3. The structure of this paper is as follows.

In Section 1 we introduce the main object of the paper - the renormalized universal trace function $F_{V_{1}, \ldots, V_{N}}(\lambda, \mu) \in\left(V_{1} \otimes \ldots \otimes V_{N}\right)[0] \otimes\left(V_{N}^{*} \otimes \ldots \otimes V_{1}^{*}\right)[0]$, where $\lambda, \mu$ are weights for $\mathfrak{g}$. It is obtained from traces of products of intertwining operators for $U_{q}(\mathfrak{g})$ weighted by an element of the maximal torus. At the end of the section we formulate the main results of the paper - Theorems 1.1-1.5.

Theorems 1.1 and 1.2 state that the function $F_{V_{1}, \ldots, V_{N}}$ satisfies two systems of difference equations, one with shifts of $\lambda$, and the other with shifts of $\mu$, which go to each other under the transformation $\lambda \rightarrow \mu, \mu \rightarrow \lambda$. In the special case $\mathfrak{g}=s l_{n}, N=1, V_{1}=S^{m n} \mathbb{C}^{n}$, these systems (as was shown in [EK1]) reduce to the trigonometric Macdonald-Ruijsenaars (MR) systems, so we call them the MR system and the dual MR system.

Theorem 1.5 states that the function $F_{V_{1}, \ldots, V_{N}}(\lambda, \mu)$ is symmetric: $F_{V_{1}, \ldots, V_{N}}(\lambda, \mu)=$ $F_{V_{N}^{*}, \ldots, V_{1}^{*}}^{*}(\mu, \lambda)$, where $*$ is the permutation of components. It follows from Theorems 1.1 and 1.2.

Theorems 1.3 and 1.4 state that the function $F_{V_{1}, \ldots, V_{n}}$ satisfies two additional systems of difference equations - the trigonometric degenerations of the qKZB and the dual qKZB equations, respectively. 
Theorems 1.1-1.5 are proved in Sections 2-5.

In Section 6 , we study the symmetry of trace functions under $q \rightarrow q^{-1}$, and define a modified trace function $u_{V}(\lambda, \mu)$, by renormalizeing $F_{V}(\lambda, \mu)$. (This function is introduced to connect our paper with the papers [FV2-FV5]; here we define it only for $N=1$, and plan to define it in general in another paper). Using the $q \rightarrow q^{-1}$ transformation properties and Theorem 1.5, we show that the function $u_{V}$ is symmetric.

In Section 7 , we compute the function $F_{V}(\lambda, \mu), u_{V}(\lambda, \mu)$ explicitly in the case $\mathfrak{g}=s l_{2}$.

In Section 8, we compute explicitly the trigonometric degeneration of the function $u$ from [FV2], in the case $N=1$. We show that this function is the same as $u_{V}(\lambda, \mu)$ up to normalization.

In Section 9, we explain that Macdonald's theory for root systems of type $A_{n-1}$ is a special case of the theory developed in this paper, for $\mathfrak{g}=s l_{n}, N=1, V_{1}=$ $S^{m n} \mathbb{C}^{n}$.

In Section 10, we consider limiting (degenerate) cases of the theory developed in this paper.

0.4. In subsequent papers of the series, we plan:

1. To give a representation theoretic-proof of the qKZB heat equation and the orthogonality relations for the trigonometric degeneration of the function $u$ ([FV2]), using the ideas of [EK1,EK2,EK3]. Cherednik's theory of difference Fourier transform and Macdonald-Mehta identities for root systems of type A is a special case of this theory, corresponding to the situation $\mathfrak{g}=s l_{n}, N=1, V_{1}=S^{m n} \mathbb{C}^{n}$.

2. To give a representation theoretic derivation of the resonance relations from [FV3] in the trigonometric case, using the ideas of [ES].

3. To generalize all the results to the case of quantum affine algebras. This involves a representation theoretic definition of the function $u$ from [FV2] for generic values of parameters, for any simple Lie algebra and representations, and a representation theoretic proof of its main properties, such as qKZB and MR equations, orthogonality, modular transformations (e.g. the qKZB heat equation). As a special case, this theory should contain Macdonald's theory for affine root systems of type $\hat{A}_{n-1}$, which was originated in [EK4] but has not been developed from an analytic standpoint. In particular, the classical limit $(q \rightarrow 1)$ of the modular transformation of the function $u$ should yield the result of Kirillov [K1,K2] which says that the modular transformation $S$ of affine Jack polynomials (which are essentially the 1-point functions of the WZW model in genus 1, see [EK4]) is given by a matrix of special values Macdonald polynomials at roots of unity.

4. Specializing this theory to the critical level, to prove that radial parts of the central elements of $U_{q}\left(\widehat{s l_{n}}\right)$ at the critical level corresponding to the representation $S^{m n} \mathbb{C}^{n}$, are elliptic Ruijsenaars operators (as far as we know, this is known only in the trigonometric degeneration).

Acknowledgments. The first author was partially supported by the NSF grant DMS-9700477, and thanks the UNC mathematics department for hospitality. The work of the first author was partly done while he was employed by the Clay Mathematics Institute as a CMI Prize Fellow. The second author was supported by the NSF grant DMS-9801582, and is grateful to the Harvard Mathematics department for hospitality. The authors are grateful to A.Kirillov Jr. for useful suggestions on how to improve the paper. 


\section{Trace FUnCtions FOR $U_{q}(\mathfrak{g})$}

\subsection{The trace functions.}

Let $\mathfrak{g}$ be a simple Lie algebra over $\mathbb{C}$. Let $\mathfrak{h}$ be a Cartan subalgebra of $\mathfrak{g}$, and $\alpha_{i}$ be simple roots of $\mathfrak{g}, i=1, \ldots, r$. Let $\left(a_{i j}\right)$ be the Cartan matrix of $\mathfrak{g}$. Let $d_{i}$ be relatively prime positive integers such that $\left(d_{i} a_{i j}\right)$ is a symmetric matrix. Let $e_{i}, f_{i}, h_{i}$ be the Chevalley generators of $\mathfrak{g}$.

Let $t$ be a complex number which is not purely imaginary, and $q=e^{t}$. For any operator $A$, we will denote $e^{t A}$ by $q^{A}$.

Let $U_{q}(\mathfrak{g})$ be the Drinfeld-Jimbo quantum group corresponding to $\mathfrak{g}$. We will use the same definition of $U_{q}(\mathfrak{g})$ as in $[\mathrm{EFK}]$. Namely $U_{q}(\mathfrak{g})$ is a Hopf algebra with generators $E_{i}, F_{i}, i=1, \ldots, r, q^{h}, h \in \mathfrak{h}$, with relations:

$$
\begin{gathered}
q^{x+y}=q^{x} q^{y}, x, y \in \mathfrak{h} \quad q^{h} E_{j} q^{-h}=q^{\alpha_{j}(h)} E_{i}, \quad q^{h} F_{j} q^{-h}=q^{-\alpha_{j}(h)} F_{i} \\
E_{i} F_{j}-F_{j} E_{i}=\delta_{i j} \frac{q^{d_{i} h_{i}}-q^{-d_{i} h_{i}}}{q^{d_{i}}-q^{-d_{i}}}, \\
\sum_{k=0}^{1-a_{i j}}(-1)^{k}\left[\begin{array}{c}
1-a_{i j} \\
k
\end{array}\right]_{q_{i}} E_{i}^{1-a_{i j}-k} E_{j} E_{i}^{k}=0, \quad i \neq j, \\
\sum_{k=0}^{1-a_{i j}}(-1)^{k}\left[\begin{array}{c}
1-a_{i j} \\
k
\end{array}\right]_{q_{i}} F_{i}^{1-a_{i j}-k} F_{j} F_{i}^{k}=0, \quad i \neq j .
\end{gathered}
$$

where $q_{i}=q^{d_{i}}$ and we used notation

$$
\left[\begin{array}{l}
n \\
k
\end{array}\right]_{q}=\frac{[n]_{q} !}{[k]_{q} ![n-k]_{q} !}, \quad[n]_{q} !=[1]_{q} \cdot[2]_{q} \cdot \ldots \cdot[n]_{q}, \quad[n]_{q}=\frac{q^{n}-q^{-n}}{q-q^{-1}}
$$

Comultiplication $\Delta$, antipode $S$ and counit $\epsilon$ in $U_{q}(\mathfrak{g})$ are given by

$$
\begin{gathered}
\Delta\left(E_{i}\right)=E_{i} \otimes q^{d_{i} h_{i}}+1 \otimes E_{i}, \quad \Delta\left(F_{i}\right)=F_{i} \otimes 1+q^{-d_{i} h_{i}} \otimes F_{i}, \quad \Delta\left(q^{h}\right)=q^{h} \otimes q^{h} \\
S\left(E_{i}\right)=-E_{i} q^{-d_{i} h_{i}}, \quad S\left(F_{i}\right)=-q^{d_{i} h_{i}} F_{i}, \quad S\left(q^{h}\right)=q^{-h} \\
\epsilon\left(E_{i}\right)=\epsilon\left(F_{i}\right)=0, \quad \epsilon\left(q^{h}\right)=1
\end{gathered}
$$

Let $M_{\mu}$ be the Verma module over $U_{q}(\mathfrak{g})$ with highest weight $\mu, v_{\mu}$ its highest weight vector. Let $V$ be a finite dimensional representation of $U_{q}(\mathfrak{g})$, and $v \in V$ a vector of weight $\mu_{v}$. It is well known that for a generic $\mu$ there exists a unique intertwining operator $\Phi_{\mu}^{v}: M_{\mu} \rightarrow M_{\mu-\mu_{v}} \otimes V$ such that $\Phi_{\mu}^{v} v_{\mu}=v_{\mu-\mu_{v}} \otimes v+$ l.o.t. (here l.o.t. denotes lower order terms). It is useful to consider the generating function of such operators, $\Phi_{\mu}^{V} \in \operatorname{Hom}_{\mathbb{C}}\left(M_{\mu}, \oplus M_{\nu} \otimes V \otimes V^{*}\right)$, defined by $\Phi_{\mu}^{V}=$ $\sum_{v \in B} \Phi_{\mu}^{v} \otimes v^{*}$, where the summation is taken over a homogeneous basis $B$ of $V$.

Let $V_{1}, \ldots, V_{N}$ be finite dimensional representations of $U_{q}(\mathfrak{g}), v_{i} \in V_{i}$ vectors of weights $\mu_{v_{i}}$, such that $\sum \mu_{v_{i}}=0$. Define the following formal series in $V_{1} \otimes \ldots \otimes V_{N}[0] \otimes q^{2(\lambda, \mu)} \mathbb{C}\left[\left[q^{-2\left(\lambda, \alpha_{1}\right)}, \ldots, q^{-2\left(\lambda, \alpha_{r}\right)}\right]\right]:$

$$
\Psi^{v_{1}, \ldots, v_{N}}(\lambda, \mu)=\left.\operatorname{Tr}\right|_{M_{\mu}}\left(\left(\Phi_{\mu-\sum_{i=2}^{N} \mu_{v_{i}}}^{v_{1}} \otimes 1^{N-1}\right) \ldots \Phi_{\mu}^{v_{N}} q^{2 \lambda}\right)
$$


It follows from Corollary 3.4, [ES] that this series converges (in a suitable region of values of the parameters) to a function of the form $q^{2(\lambda, \mu)} f(\lambda, \mu)$, where $f$ is a rational function in $q^{2\left(\lambda, \alpha_{i}\right)}$ and $q^{2\left(\mu, \alpha_{i}\right)}$, which is a finite sum of products of functions of $\lambda$ and functions of $\mu$. The function (1.1) will be called a trace function.

Define also the universal trace function, with values in $V_{1} \otimes \ldots \otimes V_{N} \otimes V_{N}^{*} \otimes \ldots \otimes V_{1}^{*}$ :

$$
\Psi_{V_{1} \ldots V_{N}}(\lambda, \mu)=\sum_{v_{i} \in B_{i}} \Psi^{v_{1}, \ldots, v_{N}}(\lambda, \mu) \otimes v_{N}^{*} \otimes \ldots \otimes v_{1}^{*}
$$

where $B_{i}$ are homogeneous bases of $V_{i}$. It is easy to see that this function takes values in $\left(V_{1} \otimes \ldots \otimes V_{N}\right)[0] \otimes\left(V_{N}^{*} \otimes \ldots \otimes V_{1}^{*}\right)[0]$.

Using the generating functions $\Phi_{\mu}^{V}$, one can express the universal trace function as follows:

$$
\Psi_{V_{1} \ldots V_{N}}(\lambda, \mu)=\operatorname{Tr}\left(\left(\Phi_{\mu+\sum_{i=2}^{N} h_{1}(* i)}^{V_{1}} \otimes 1^{N-1}\right) \ldots \Phi_{\mu}^{V_{N}} q^{2 \lambda}\right),
$$

where we label the components $V_{i}$ by $i$ and $V_{i}^{*}$ by $* i$, and the notation $h^{(k)}$ for a label $k$ was defined in [F]: when acting on a homogeneous multivector, $h^{(k)}$ has to be replaced with the weight in the k-th component.

Example 1. (See Section 7) Let $\mathfrak{g}=s l_{2}$. In this case let us represent weights by complex numbers, so that the unique fundamental weight corresponds to 1 . If $N=1$, and $V=V_{1}$ is the 3 -dimensional representation, then

$$
\Psi_{V}(\lambda, \mu)=\frac{q^{\lambda \mu}}{1-q^{-2 \lambda}}\left(1+\left(q^{2}-q^{-2}\right) \frac{q^{-2 \lambda}}{\left(1-q^{2 \mu}\right)\left(1-q^{-2(\lambda-1)}\right)}\right)
$$

(since $V[0]$ is 1-dimensional, we view the function $\Psi_{V}$ as a scalar function). This example is also computed in [ES1].

\subsection{The main results.}

It turns out that the trace function $\Psi_{V_{1} \ldots V_{N}}(\lambda, \mu)$ satisfies some remarkable difference equations. These equations are written in terms of so called dynamical R-matrices. Below we give a brief introduction to the theory of dynamical Rmatrices, referring the reader to the expository paper [ESch] for a more detailed discussion of them.

Let $V, W$ be finite dimensional representations of $U_{q}(\mathfrak{g})$. Recall from [EV] the definitions of the fusion matrix and the exchange matrix.

The fusion matrix is the operator $J_{W V}(\mu): W \otimes V \rightarrow W \otimes V$ defined by the formula

$$
\left(\Phi_{\mu-\mu_{v}}^{w} \otimes 1\right) \Phi_{\mu}^{v}=\Phi_{\mu}^{J_{W V}(\mu)(w \otimes v)} .
$$

The exchange matrix $R_{V W}(\mu) \in \operatorname{End}(V \otimes W)$ is defined by

$$
R_{V W}(\mu)=\left.J_{V W}^{-1}(\mu) \mathcal{R}^{21}\right|_{V \otimes W} J_{W V}^{21}(\mu),
$$

where $\mathcal{R}$ is the universal R-matrix of $U_{q}(\mathfrak{g})$.

We will also use the universal fusion matrix $J(\lambda)$ and the universal exchange matrix $R(\lambda)$. They take values in a completion of $U_{q}(\mathfrak{g}) \otimes U_{q}(\mathfrak{g})$ and are defined by 
the condition that they give $J_{V W}(\lambda), R_{V W}(\lambda)$ when evaluated in representations $V, W$ (cf. also [ABRR],[JKOS]).

Remark. The fusion matrix describes how to "fuse" together two intertwining operators. The exchange matrix describes how to exchange the order of intertwining operators. The fusion matrix satisfies the 2-cocycle identity (see below), and is sometimes referred to as a "quasi-Hopf twist". The exchange matrix satisfies the quantum dynamical Yang-Baxter equation, and is refereed to as a "dynamical Rmatrix".

Let $\mathbb{J}(\lambda):=J(-\lambda-\rho)$, where $\rho$ is the half-sum of positive roots, and let $\mathbb{R}(\lambda)=$ $R(-\lambda-\rho)$. Let $\mathbb{Q}(\lambda)=m_{21}\left(1 \otimes S^{-1}\right)(\mathbb{J}(\lambda))$, where $m_{21}(a \otimes b):=b a$, and $S$ is the antipode. It is easy to show that $\mathbb{Q}(\lambda)$ is invertible for generic $\lambda$.

Define

$$
\mathbb{J}^{1 \ldots N}(\lambda)=\mathbb{J}^{1,2 \ldots N}(\lambda) \mathbb{J}^{2,3 \ldots N}(\lambda) \ldots \mathbb{J}^{N-1, N}(\lambda),
$$

where, for example, $\mathbb{J}^{1,2 \ldots N}$ stands for $\left(1 \otimes \Delta_{n-1}\right)(\mathbb{J})$, where $\Delta_{n-1}: U_{q}(\mathfrak{g}) \rightarrow$ $U_{q}(\mathfrak{g})^{n-1}$ is the iterated coproduct. We agree that $\mathbb{J}^{1}(\lambda)=1$. Thus $\mathbb{J}^{1 \cdots N}(\lambda)$ describes how to "fuse" $N$ intertwining operators.

Let

$$
\delta_{q}(\lambda)=q^{2(\lambda, \rho)} \prod_{\alpha>0}\left(1-q^{-2(\lambda, \alpha)}\right)
$$

be the Weyl denominator.

Set

$$
\varphi_{V_{1} \ldots V_{N}}(\lambda, \mu)=\mathbb{J}^{1 \ldots N}(\lambda)^{-1} \Psi_{V_{1} \ldots V_{N}}(\lambda, \mu) \delta_{q}(\lambda) .
$$

Finally, introduce the renormalized trace function

$$
F_{V_{1} \ldots V_{n}}(\lambda, \mu)=\left[\mathbb{Q}^{-1}(\mu)^{(* N)} \otimes \ldots \otimes \mathbb{Q}^{-1}\left(\mu-h^{(* 2 \ldots * N)}\right)^{(* 1)}\right] \varphi_{V_{1} \ldots V_{N}}(\lambda,-\mu-\rho) .
$$

It is convenient to formulate properties of trace functions using this renormalization.

Example 2. (See Section 7) If $\mathfrak{g}=s l_{2}, N=1$, and $V=V_{1}$ is the 3-dimensional representation, then

$$
F_{V}(\lambda, \mu)=q^{-\lambda \mu} \frac{q^{2(\lambda+\mu)}-q^{2 \lambda-2}-q^{2 \mu-2}+1}{\left(1-q^{2 \lambda-2}\right)\left(1-q^{2 \mu-2}\right)} .
$$

(This formula is obtained after simplifications from formula (7.20) below when $m=1$ ). Note that it is seen from this formula that $F_{V}$ is symmetric in $\lambda$ and $\mu$.

The following theorems describe the properties of $F_{V_{1} \ldots V_{N}}(\lambda, \mu)$.

For any finite dimensional $U_{q}(\mathfrak{g})$-module $W$, define the difference operator $\mathcal{D}_{W}$ acting on functions of $\lambda \in \mathfrak{h}^{*}$ with values in $\left(V_{1} \otimes \ldots \otimes V_{N}\right)[0]$ given by the formula

$$
\mathcal{D}_{W}=\left.\sum_{\nu} \operatorname{Tr}\right|_{W[\nu]}\left(\mathbb{R}_{W V_{1}}^{01}\left(\lambda+h^{(2 \ldots N)}\right) \ldots \mathbb{R}_{W V_{N}}^{0 N}(\lambda)\right) T_{\nu}
$$

where $T_{\nu} f(\lambda)=f(\lambda+\nu)$, and the component $W$ is labeled by 0 . 
Theorem 1.1. (Macdonald-Ruijsenaars equations)

$$
\mathcal{D}_{W}^{\lambda} F_{V_{1} \ldots V_{N}}(\lambda, \mu)=\chi_{W}\left(q^{-2 \mu}\right) F_{V_{1} \ldots V_{N}}(\lambda, \mu),
$$

where $\chi_{W}(x)=\sum \operatorname{dim} W[\nu] x^{\nu}$ is the character of $W$, and by $\mathcal{D}_{W}^{\lambda}$ we mean the operator $\mathcal{D}_{W}$ acting on $F$ as a function of $\lambda$, in components $V_{1}, \ldots, V_{N}$.

Theorem 1.1 is proved in Section 2.

Example 3. If $\mathfrak{g}=s l_{2}, N=1$, and $V=V_{1}$ is the 3-dimensional representation, and $W$ is the 2-dimensional representation, then

$$
\mathcal{D}_{W}=T+\frac{\left(1-q^{2 \lambda-4}\right)\left(1-q^{2 \lambda+2}\right)}{\left(1-q^{2 \lambda-2}\right)\left(1-q^{2 \lambda}\right)} T^{-1},
$$

where $T f(\lambda)=f(\lambda+1)$. To prove this, it is enough to check that this operator is the unique operator of the form $a(\lambda) T+b(\lambda) T^{-1}$ such that (1.11) holds for the function $F_{V}$ given above (note that in our case $\chi_{W}\left(q^{-2 \mu}\right)=q^{\mu}+q^{-\mu}$ ).

Introduce also the dual Macdonald-Ruijsenaars operators $\mathcal{D}_{W}^{\vee}$, acting on functions of $\mu \in \mathfrak{h}^{*}$ with values in $\left(V_{N}^{*} \otimes \ldots \otimes V_{1}^{*}\right)[0]$, by the formula

$$
\mathcal{D}_{W}^{\vee}=\left.\sum_{\nu} \operatorname{Tr}\right|_{W[\nu]}\left(\mathbb{R}_{W V_{N}^{*}}^{01}\left(\mu+h^{(* 1 \ldots * N-1)}\right) \ldots \mathbb{R}_{W V_{1}^{*}}^{0 N}(\mu)\right) T_{\nu},
$$

where $V_{j}^{*}$ is considered as a module over $U_{q}(\mathfrak{g})$ via the antipode.

Theorem 1.2. (Dual Macdonald-Ruijsenaars equations)

$$
\mathcal{D}_{W}^{\vee, \mu} F_{V_{1} \ldots V_{N}}(\lambda, \mu)=\chi_{W}\left(q^{-2 \lambda}\right) F_{V_{1} \ldots V_{N}}(\lambda, \mu),
$$

where $\mathcal{D}_{W}^{\vee, \mu}$ is $\mathcal{D}_{W}^{\vee}$ acting on $F$ as a function of $\mu$, in components $V_{N}^{*}, \ldots, V_{1}^{*}$.

Theorem 1.2 is proved in Section 3 .

To formulate the next two results, we need to define some operators acting on functions of $\lambda$ and $\mu$ with values in $\left(V_{1} \otimes \ldots \otimes V_{N}\right)[0] \otimes\left(V_{N}^{*} \otimes \ldots \otimes V_{1}^{*}\right)[0]$.

For $j=1, \ldots, N$ define the operators

$$
D_{j}=\left(q^{-2 \mu-\sum x_{i}^{2}}\right)_{* j}\left(q^{-2 \sum x_{i} \otimes x_{i}}\right)_{* j, * 1 \ldots * j-1},
$$

where $x_{i}$ is an orthonormal basis of $\mathfrak{h}$. Also, define the operators

$$
K_{j}=
$$

$\mathbb{R}_{j+1, j}\left(\lambda+h^{(j+2 \ldots N)}\right)^{-1} \ldots \mathbb{R}_{N j}(\lambda)^{-1} \Gamma_{j} \mathbb{R}_{j 1}\left(\lambda+h^{(2 \ldots j-1)}+h^{(j+1 \ldots N)}\right) \ldots \mathbb{R}_{j j-1}\left(\lambda+h^{(j+1 \ldots N)}\right)$,

where $\Gamma_{j} f(\lambda):=f\left(\lambda+h^{(j)}\right)$, and $h^{j \ldots k}$ acting on a homogeneous multivector has to be replaced with the sum of weights of components $j, \ldots, k$ of this multivector. It is easy to check that $D_{j}$ commute with each other, and its is known that so do $K_{j}([\mathrm{~F}])$.

Remark. As we have mentioned before, these operators are the trigonometric limits of the qKZB operators with spectral parameters, introduced by Felder.

Analogously, define the operators

$$
D_{j}^{\vee}=\left(q^{-2 \lambda-\sum x_{i}^{2}}\right)_{j}\left(q^{-2 \sum x_{i} \otimes x_{i}}\right)_{j, j+1 \ldots N},
$$

and

$$
K_{j}^{\vee}=\mathbb{R}_{* j-1, * j}\left(\mu+h^{(* 1 \ldots * j-2)}\right)^{-1} \ldots \mathbb{R}_{* 1, * j}(\mu)^{-1} \Gamma_{* j} \times
$$$$
\text { where } \Gamma_{* j} f(\mu)=f\left(\mu+h^{* j}\right) \text {. Like } D_{j}, K_{j} \text {, the operators } D_{j}^{\vee}, K_{j}^{\vee} \text { commute. }
$$ 
Theorem 1.3. (the $q K Z B$ equations) The function $F_{V_{1} \ldots V_{N}}$ satisfies the $q K Z B$ equations:

$$
F_{V_{1} \ldots V_{N}}(\lambda, \mu)=\left(K_{j} \otimes D_{j}\right) F_{V_{1} \ldots V_{N}}(\lambda, \mu) .
$$

Theorem 1.4. (the dual qKZB equations)

$$
F_{V_{1} \ldots V_{N}}(\lambda, \mu)=\left(D_{j}^{\vee} \otimes K_{j}^{\vee}\right) F_{V_{1} \ldots V_{N}}(\lambda, \mu) .
$$

Example 4. Let $\mathfrak{g}=s l_{2}, N=2, V_{1}=V_{2}=\mathbb{C}^{2}$ with standard basis $v_{+}, v_{-}$. In this case $V_{1} \otimes V_{2}[0]$ is 2-dimensional with basis $v_{+} \otimes v_{-}, v_{-} \otimes v_{+}$, and the action of the dynamical R-matrix in this basis is

$$
\mathbb{R}(\lambda)=\left(\begin{array}{cc}
1 & q^{-1 / 2} \frac{q^{-1}-q}{q^{-2 \lambda}-1} \\
\frac{q^{-1}-q}{q^{2 \lambda}-1} & \frac{\left(q^{-2 \lambda}-q^{2}\right)\left(q^{-2 \lambda}-q^{-2}\right)}{\left(q^{-2 \lambda}-1\right)^{2}}
\end{array}\right) .
$$

Therefore, if $F_{V_{1}, V_{2}}(\lambda, \mu)$ is represented by a 2 by 2 matrix with respect to the above basis then the qKZB equation corresponding to $j=2$ has the form

$$
\begin{gathered}
\left(\begin{array}{cc}
\frac{\left(q^{-2 \lambda}-q^{2}\right)\left(q^{-2 \lambda}-q^{-2}\right)}{\left(q^{-2 \lambda}-1\right)^{2}} & \frac{q^{-1}-q}{q^{-2 \lambda}-1} \\
\frac{q^{-1}-q}{q^{2 \lambda}-1} & 1
\end{array}\right)\left(\begin{array}{cc}
F_{11}(\lambda, \mu) & F_{12}(\lambda, \mu) \\
F_{21}(\lambda, \mu) & F_{22}(\lambda, \mu)
\end{array}\right)\left(\begin{array}{cc}
q^{\mu} & 0 \\
0 & q^{-\mu}
\end{array}\right)= \\
\left(\begin{array}{cc}
F_{11}(\lambda+1, \mu) & F_{12}(\lambda-1, \mu) \\
F_{21}(\lambda+1, \mu) & F_{22}(\lambda-1, \mu)
\end{array}\right)
\end{gathered}
$$

Here for convenience we took the shift operator $\Gamma_{2}$ from the left side of the equation to the right side.

Remark 1. We should warn the reader that the term "qKZB equations" is normally used for equations which contain elliptic dynamical R-matrices with spectral parameters, and are difference equations with respect to these parameters $z_{1}, \ldots, z_{N}$ (see e.g.[FTV1]). The equations we consider are a limiting case of the "genuine" qKZB equations, when the modular parameter $\tau$ goes to infinity, and the ratios of the spectral parameters $z_{j} / z_{j+1}$ go to zero, with $e^{-2 \pi \operatorname{Im} \tau}<<\left|z_{j} / z_{j+1}\right|<<1$. Namely, the equations considered here are the equations satisfied by the limit (if it exists) of a solution of the "genuine" qKZB equations in the described asymptotic zone. Throughout this paper, we will abuse terminology and use the term "qKZB equations" to refer to this limiting case.

Remark 2. If $N=1$, equations (1.18) and (1.19) are trivial: (1.18) says that the $V$-component of $F_{V}$ has zero weight, and (1.19) says that the $V^{*}$-component of $F_{V}$ has zero weight.

Remark 3. It is not hard to show using the quantum dynamical Yang-Baxter equation for the dynamical R-matrices that the Macdonald-Ruijsenaars operators commute with the qKZB operators. Similarly, the dual Macdonald-Ruijsenaars operators commute with the dual qKZB operators.

Remark 4. Theorems 1.1, 1.3 have the following interpretation. Suppose $v_{1}, \ldots, v_{N}$ are homogeneous vectors in $V_{1}, \ldots, V_{N}$ of weights $\nu_{1}, \ldots, \nu_{N}, \sum \nu_{i}=0$. Then the function $\left(F_{V_{1}, \ldots, V_{N}}(\lambda, \mu), v_{1} \otimes \ldots \otimes v_{N}\right)$ is an common eigenfunction of the operators $\mathcal{D}_{W}^{\lambda}$ and $K_{j}$ with eigenvalues equal to $\chi_{W}\left(q^{-2 \mu}\right)$ and 
$\Lambda_{j}(\mu)=q^{-2\left(\mu, \nu_{j}\right)+\left(\nu_{j}, \nu_{j}\right)+2 \sum_{i<j}\left(\nu_{i}, \nu_{j}\right)}$, respectively. Thus the trace functions provide a solution of the problem of simultaneous diagonalization of the MacdonaldRuijsenaars operators $\mathcal{D}_{W}^{\lambda}$ and qKZB operators $K_{j}$.

Remark 5. The problem to deduce equations of type (1.18) for trace functions (in the case of affine Lie algebras) was suggested to the first author in 1992 by his adviser Igor Frenkel, as a topic of his Ph.D. thesis. However, the first author failed to solve this problem at that time, partly because the adequate framework, the theory of dynamical R-matrices, was not around yet.

Theorem 1.5. (the symmetry identities) The function $F_{V_{1} \ldots V_{N}}$ is symmetric:

$$
F_{V_{1} \ldots V_{N}}(\lambda, \mu)=F_{V_{N}^{*} \ldots V_{1}^{*}}^{*}(\mu, \lambda),
$$

where $F^{*}$ is the result of interchanging the factors $\left(V_{1} \otimes \ldots \otimes V_{N}\right)[0]$ and $\left(V_{N}^{*} \otimes \ldots \otimes V_{1}^{*}\right)[0]$.

Theorem 1.4 is proved in Section 4. Theorem 1.5 is proved in Section 5, using Theorems 1.1 and 1.2. Theorem 1.5 and Theorem 1.4 obviously imply Theorem 1.3 .

Remark 1. Theorem 1.3 can also be derived directly, using the method of Frenkel-Reshetikhin of derivation of the Knizhnik-Zamolodchikov equations. However, this derivation is rather long, and we don't give it here.

Remark 2. In the special case $\mathfrak{g}=s l_{n}, N=1, V_{1}=S^{m n} \mathbb{C}^{n}$, the function $F$ is closely related to the kernel of Cherednik's difference Fourier transform for $s l_{n}([\mathrm{C}]$, and the symmetry theorem above (Theorem 1.5) is closely related to Cherednik's theorem on the symmetry of the difference Fourier transform $[\mathrm{C}]$.

Remark 3. In the special case of Remark 2, Theorem 1.5 was proved in [ES1] (Theorem 5.6).

Remark 4. Theorems 1.1-1.5 can be generalized to the case when $\mathfrak{g}$ is any symmetrizable Kac-Moody algebra, and $V_{i}$ highest weight modules over $U_{q}(\mathfrak{g})$. In this case, the functions $\Psi, F$ make sense as formal power series, and (if $\mathfrak{g}$ is not finite dimensional) the operators $\mathcal{D}_{W}, \mathcal{D}_{W}^{\vee}$ are infinite difference operators: they are infinite sums of terms $f(\lambda) T_{\nu}$. However, one can show that these sums make sense as operators on power series. We plan to discuss this elsewhere.

\section{The Macdonald-Ruijsenaars (MR) equations}

\subsection{Radial parts.}

Let $V$ be a finite dimensional $U_{q}(\mathfrak{g})$ module. Then we have the following proposition.

Proposition 2.1. (i) For any element $X$ of $U_{q}(\mathfrak{g})$ there exists a unique difference operator $D_{X}$ acting on $V[0]$-valued functions, such that

$$
\operatorname{Tr}\left(\Phi_{\mu}^{V} X q^{2 \lambda}\right)=D_{X} \operatorname{Tr}\left(\Phi_{\mu}^{V} q^{2 \lambda}\right) .
$$

(ii) If $X$ is central then $D_{X Y}=D_{Y} D_{X}$ for all $Y \in U_{q}(\mathfrak{g})$. In particular, if $X$, $Y$ are central then $D_{X} D_{Y}=D_{Y} D_{X}$.

Proof. This is proved in Section 6 of [EK1] (the assumption $g=g l_{n}$, which is made throughout [EK1], is not important for the proof of this result). 
We will call the operator $D_{X}$ the radial part of $X$.

Now recall the Drinfeld-Reshetikhin construction of central elements of $U_{q}(\mathfrak{g})$ $([\mathrm{D}, \mathrm{R}])$. In this construction, one defines elements $C_{W}$ corresponding to finite dimensional representations $W$ of $U_{q}(\mathfrak{g})$ by the formula

$$
C_{W}=\left.\operatorname{Tr}\right|_{W}\left(1 \otimes \pi_{W}\right)\left(\mathcal{R}^{21} \mathcal{R}\left(1 \otimes q^{2 \rho}\right)\right) .
$$

The map $W \rightarrow C_{W}$ defines a homomorphism of the Grothendieck ring of the category of finite dimensional representations of $U_{q}(\mathfrak{g})$ to the center of $U_{q}(\mathfrak{g})$.

Define difference operators $\mathcal{M}_{W}:=D_{C_{W}}$.

Proposition 2.2. (i) $\mathcal{M}_{W} \mathcal{M}_{U}=\mathcal{M}_{U} \mathcal{M}_{W}=\mathcal{M}_{W \otimes U}$.

(ii) $\mathcal{M}_{W} \Psi_{V}(\lambda, \mu)=\chi_{W}\left(q^{2(\mu+\rho)}\right) \Psi_{V}(\lambda, \mu)$, where $\chi_{W}(x)=\sum_{\nu} \operatorname{dim} W[\nu] x^{\nu}$ is the character of $W$.

Proof. See [EK1] (the proof for $g l(n)$ generalizes tautologically to other Lie algebras).

This proposition shows that if we can compute $\mathcal{M}_{W}$ explicitly then we will get a system of difference equations for $\Psi_{V}$.

\subsection{The difference equations.}

Let $G(\lambda)=q^{-2 \rho} \mathbb{Q}^{-1}(\lambda) S(\mathbb{Q})(\lambda-h)$.

Theorem 2.3. For any $V[0]$-valued function on $\mathfrak{h}^{*}$,

$$
\left(\mathcal{M}_{W} f\right)(\lambda)=\left.\sum_{\nu} \operatorname{Tr}\right|_{W[\nu]}\left(G(\lambda+h) \mathbb{R}_{W V}(\lambda)\right) f(\lambda+\nu) .
$$

The proof of Theorem 2.3 occupies Sections 2.3-2.6.

\subsection{The defining property of $J$.}

Define

$$
\mathcal{J}(\lambda):=J\left(-\lambda-\rho+\frac{1}{2}\left(h^{(1)}+h^{(2)}\right)\right) .
$$

Lemma 2.4. (The Arnaudon-Buffenoir-Ragoucy-Roche equation, [ABRR], see also [JKOS] $)$ Let $V, W$ be finite dimensional $U_{q}(\mathfrak{g})$-modules. Then

$$
\mathcal{R}^{21}\left(q^{2 \lambda}\right)_{1} \mathcal{J}(\lambda)=\mathcal{J}(\lambda) q^{\sum x_{i} \otimes x_{i}}\left(q^{2 \lambda}\right)_{1} .
$$

Moreover, this solution is unique among solutions of the form $1+N$, where $N$ has only summands with positive weight of the second component.

Remark. As pointed out in [EV], this equation is a limiting case of the quantum Knizhnik-Zamolodchikov equation of [FR], obtained when the affine quantum group degenerates into the finite dimensional quantum group.

Proof. We recall ([ABRR], see also $[\mathrm{EV}]$, section 9) that $J_{W V}(\lambda)$ satisfies the equation

$$
J_{W V}(\lambda)\left(q^{2(\lambda+\rho)-\sum x_{i}^{2}}\right)_{2}=\mathcal{R}_{V W}^{21} q^{-\sum x_{i} \otimes x_{i}}\left(q^{2(\lambda+\rho)-\sum x_{i}^{2}}\right)_{2} J_{W V}(\lambda),
$$


Using the weight zero property of $J$, we get

$$
J_{W V}(\lambda)\left(q^{-2(\lambda+\rho)}\right)_{1}\left(q^{-\sum x_{i}^{2}}\right)_{2}=\mathcal{R}_{V W}^{21}\left(q^{-2(\lambda+\rho)}\right)_{1} q^{-\sum\left(x_{i} \otimes 1+1 \otimes x_{i}\right)\left(1 \otimes x_{i}\right)} J_{W V}(\lambda),
$$

Now apply both sides of (2.7) to the subspace $W[\nu] \otimes V[\mu]$. Using the weight zero condition again, we get (on that subspace)

$$
J_{W V}(\lambda)\left(q^{-2(\lambda+\rho)}\right)_{1} q_{1}^{\mu+\nu}\left(q^{\nu}\right)_{2}=\mathcal{R}_{V W}^{21}\left(q^{-2(\lambda+\rho)}\right)_{1}\left(q^{\mu+\nu}\right)_{1} J_{W V}(\lambda)
$$

Thus, replacing $\lambda$ with $\lambda+\frac{1}{2}(\mu+\nu)$, we get

$$
J_{W V}\left(\lambda+\frac{1}{2}(\mu+\nu)\right)\left(q^{-2(\lambda+\rho)}\right)_{1}\left(q^{\nu}\right)_{2}=\mathcal{R}_{V W}^{21}\left(q^{-2(\lambda+\rho)}\right)_{1} J_{W V}\left(\lambda+\frac{1}{2}(\mu+\nu)\right)
$$

which means that

$\mathcal{R}_{V W}^{21}\left(q^{-2(\lambda+\rho)}\right)_{1} J_{W V}\left(\lambda+\frac{1}{2}\left(h^{(1)}+h^{(2)}\right)\right)=J_{W V}\left(\lambda+\frac{1}{2}\left(h^{(1)}+h^{(2)}\right)\right) q^{\sum x_{i} \otimes x_{i}}\left(q^{-2(\lambda+\rho)}\right)_{1}$,

This implies the first statement. The second statement is straightforward, and also follows from $[\mathrm{ABRR}]$.

We will also need the cocycle identity for $\mathcal{J}$. To deduce it, recall the 2 -cocycle identity for $J$ (see $[\mathrm{A}, \mathrm{EV}])$ :

$$
J^{12,3}(\lambda) J^{12}\left(\lambda-h^{(3)}\right)=J^{1,23}(\lambda) J^{23}(\lambda)
$$

Thus the cocycle identity for $\mathcal{J}$ has the form

$$
\mathcal{J}^{12,3}(\lambda) \mathcal{J}^{12}\left(\lambda+\frac{1}{2} h^{(3)}\right)=\mathcal{J}^{1,23}(\lambda) \mathcal{J}^{23}\left(\lambda-\frac{1}{2} h^{(1)}\right)
$$

2.4. The function $Z_{V}$. Let $V$ be a finite-dimensional $U_{q}(\mathfrak{g})$-module. Consider the following function with values in $V \otimes V^{*} \otimes U_{q}(\mathfrak{g})$ (with components labeled as $1, * 1,2)$ :

$$
Z_{V}(\lambda, \mu)=\operatorname{Tr}_{0}\left(\Phi_{\mu}^{V, 01} \mathcal{R}^{20} q^{2 \lambda}\right)
$$

\section{Lemma 2.5.}

$$
Z_{V}(\lambda, \mu)=\mathcal{R}^{21}\left(q^{2 \lambda}\right)_{1} Z_{V}(\lambda, \mu)
$$

Proof. Let us move the R-matrix $\mathcal{R}^{20}$ around the trace. We get

$$
\begin{gathered}
Z_{V}(\lambda, \mu)=\operatorname{Tr}_{0}\left(\mathcal{R}^{21} \mathcal{R}^{20} \Phi_{\mu}^{V, 01} q^{2 \lambda}\right)= \\
\mathcal{R}^{21} \operatorname{Tr}_{0}\left(\Phi_{\mu}^{V, 01} q^{2 \lambda} \mathcal{R}^{20}\right),
\end{gathered}
$$

and the Lemma follows after interchangicng $q^{2 \lambda}$ with $\Phi_{\mu}^{V}$ and moving it around the trace. 


\section{Corollary 2.6.}

$$
Z_{V}(\lambda, \mu)=\mathcal{J}^{12}(\lambda) \Psi_{V}^{(1)}\left(\lambda+\frac{1}{2} h^{(2)}, \mu\right)
$$

Proof. Both sides of the equation satisfy $(2.14)$ and have the form $\Psi_{V}^{(1)}\left(\lambda+\frac{1}{2} h^{(2)}, \mu\right)+$ $N$, where $N$ has positive degree in component 2 . It is easy to show that a solution of (2.14) with such property is unique, which implies the lemma.

\subsection{The function $X_{V}$.}

Define the function

$$
X_{V}(\lambda, \mu)=\operatorname{Tr}_{0}\left(\Phi_{\mu}^{V, 01} \mathcal{R}^{20} q^{2 \lambda}\left(\mathcal{R}^{03}\right)^{-1}\right),
$$

with values in $V \otimes V^{*} \otimes U_{q}(\mathfrak{g}) \otimes U_{q}(\mathfrak{g})$ (components labeled $\left.1, * 1,2,3\right)$.

\section{Lemma 2.7.}

$$
X_{V}(\lambda, \mu)=\mathcal{R}^{12,3}\left(q^{2 \lambda}\right)_{3} X_{V}(\lambda, \mu)\left(q^{-2 \lambda}\right)_{3}\left(\mathcal{R}^{23}\right)^{-1} .
$$

Proof. We have

$$
\begin{gathered}
X_{V}(\lambda, \mu)=\operatorname{Tr}_{0}\left(\left(\mathcal{R}^{03}\right)^{-1} \Phi_{\mu}^{V, 01} \mathcal{R}^{20} q^{2 \lambda}\right)= \\
\mathcal{R}^{13} \operatorname{Tr}_{0}\left(\Phi_{\mu}^{V, 01}\left(\mathcal{R}^{03}\right)^{-1} \mathcal{R}^{20} q^{2 \lambda}\right)= \\
\mathcal{R}^{13} \mathcal{R}^{23} \operatorname{Tr}_{0}\left(\Phi_{\mu}^{V, 01} \mathcal{R}^{20}\left(\mathcal{R}^{03}\right)^{-1} q^{2 \lambda}\right)\left(\mathcal{R}^{23}\right)^{-1}= \\
\mathcal{R}^{13} \mathcal{R}^{23}\left(q^{2 \lambda}\right)_{3} X_{V}(\lambda, \mu)\left(q^{-2 \lambda}\right)_{3}\left(\mathcal{R}^{23}\right)^{-1}
\end{gathered}
$$

The lemma is proved.

\section{Lemma 2.8.}

$$
X_{V}(\lambda, \mu)=\mathcal{J}^{3,12}(\lambda) \mathcal{J}^{12}\left(\lambda-\frac{1}{2} h^{(3)}\right) \Psi_{V}^{(1)}\left(\lambda+\frac{1}{2}\left(h^{(2)}-h^{(3)}\right), \mu\right) \mathcal{J}^{32}(\lambda)^{-1}
$$

Proof. Consider the function $X(\lambda)=\mathcal{J}^{3,12}(\lambda)^{-1} X_{V}(\lambda) \mathcal{J}^{32}(\lambda)$. (for brevity we suppress the dependence on $\mu$ in the notation). By Lemma 2.7 and Lemma 2.4, this function satisfies the equation

$$
\left(q^{2 \lambda}\right)_{3} q^{\sum\left(x_{i} \otimes 1 \otimes x_{i}+1 \otimes x_{i} \otimes x_{i}\right)} X(\lambda)=X(\lambda)\left(q^{2 \lambda}\right)_{3} q^{\sum 1 \otimes x_{i} \otimes x_{i}} .
$$

On the other hand, by the definition of $X_{V}$, the function $X$ is of the form $Z_{V}\left(\lambda-\frac{1}{2} h^{(3)}\right)+N$, where $N$ has negative weights in the third component. As before, a solution of (2.21) with such property is unique, so it must coincide with its highest term. Thus, $N=0$, and by Corollary 2.6 we get the Lemma.

Corollary 2.9.

$$
\operatorname{Tr}_{0}\left(\mathcal{R}^{20}\left(\mathcal{R}^{03}\right)^{-1} \Phi_{\mu}^{V, 01} q^{2 \lambda}\right)=
$$

$$
\left(q^{-2 \lambda}\right)_{2} \mathcal{J}^{3,12}(\lambda) \mathcal{J}^{12}\left(\lambda-\frac{1}{2} h^{(3)}\right) \Psi_{V}^{(1)}\left(\lambda+\frac{1}{2}\left(h^{(2)}-h^{(3)}\right), \mu\right) \mathcal{J}^{32}(\lambda)^{-1}\left(q^{2 \lambda}\right)_{2} .
$$

Proof. Straightforward from Lemma 2.8. 


\subsection{The difference operators.}

To obtain the action of the operators $\mathcal{M}_{W}$ on traces, we need to apply to both sides of $(2.22)$ the operation $m_{23}(1 \otimes 1 \otimes S)$, then multiply by $q^{2 \rho}$ and take the trace.

Let $\mathcal{R}=\sum a_{i} \otimes b_{i}, \mathcal{R}^{-1}=\sum a_{i}^{\prime} \otimes b_{i}^{\prime}$. Let $u=\sum S\left(b_{i}\right) a_{i}$ be the Drinfeld element. Let $\mathcal{J}(\lambda)=\sum c_{k} \otimes d_{k}(\lambda)$, and $\mathcal{J}^{-1}(\lambda)=\sum c_{k}^{\prime} \otimes d_{k}^{\prime}(\lambda)$. Then we get

$$
\mathcal{M}_{W} \Psi_{V}(\lambda, \mu)=
$$

$\operatorname{Tr}_{2} \sum\left[d_{i}^{(1)}(\lambda) c_{j} \otimes q^{-2 \lambda} d_{i}^{(2)}(\lambda) d_{j}\left(\lambda+\frac{1}{2} h^{(2)}\right)\right] \Psi_{V}\left(\lambda+h^{(2)}, \mu\right)\left(d_{k}^{\prime}(\lambda) q^{2 \lambda} S\left(c_{k}^{\prime}\right) S\left(c_{i}\right) q^{2 \rho}\right)_{2}$.

Let us simplify the expression for $\sum d_{k}^{\prime}(\lambda) q^{2 \lambda} S\left(c_{k}^{\prime}\right)$, which enters in (2.23).

\section{Lemma 2.10.}

$$
\sum d_{k}^{\prime}(\lambda) q^{2 \lambda} S\left(c_{k}^{\prime}\right)=q^{\sum x_{i}^{2}} P(\lambda) S(u) q^{2 \lambda},
$$

where $P(\lambda):=\sum d_{i}^{\prime}(\lambda) S^{-1}\left(c_{i}^{\prime}\right)$.

Proof. From Lemma 2.4 we get

$$
\mathcal{J}^{-1}(\lambda)\left(q^{-2 \lambda}\right)_{1}=q^{-\sum x_{i} \otimes x_{i}}\left(q^{-2 \lambda}\right)_{1} \mathcal{J}^{-1}(\lambda) \mathcal{R}^{21} .
$$

Applying $m_{21}(S \otimes 1)$ on both sides, we get

$$
\sum d_{k}^{\prime}(\lambda) q^{2 \lambda} S\left(c_{k}^{\prime}\right)=q^{\sum x_{i}^{2}} \sum d_{i}^{\prime}(\lambda) a_{i} S\left(b_{i}\right) S\left(c_{i}^{\prime}\right) q^{2 \lambda},
$$

which implies the Lemma, since $\sum a_{i} S\left(b_{i}\right)=S(u)$.

Substituting (2.24) into (2.23) and using the cyclic property of the trace, we get

$$
\begin{gathered}
\mathcal{M}_{W} \Psi_{V}(\lambda, \mu)= \\
\sum d_{i}^{(1)}(\lambda) c_{j} \Psi_{V}(\lambda+\nu, \mu) \operatorname{Tr}_{2}\left[q^{\sum x_{i}^{2}} P(\lambda) S(u) q^{2 \lambda} S\left(c_{i}\right) q^{2 \rho} q^{-2 \lambda} d_{i}^{(2)}(\lambda) d_{j}\left(\lambda+\frac{1}{2} \nu\right)\right]= \\
\sum d_{i}^{(1)}(\lambda) c_{j} \Psi_{V}(\lambda+\nu, \mu) \operatorname{Tr}_{2}\left[q^{\sum x_{i}^{2}} P(\lambda) q^{2 \lambda} S^{-1}\left(c_{i}\right) q^{-2 \lambda} d_{i}^{(2)}(\lambda) d_{j}\left(\lambda+\frac{1}{2} \nu\right) S(u) q^{2 \rho}\right]
\end{gathered}
$$

Lemma 2.11 .

$$
\begin{gathered}
\sum d_{i}^{(1)}(\lambda) \otimes q^{2 \lambda} S^{-1}\left(c_{i}\right) q^{-2 \lambda} d_{i}^{(2)}(\lambda)= \\
\sum\left(a_{j}^{\prime(1)} d_{k}^{(1)}(\lambda)\right)_{1} q^{-\sum\left(x_{i}\right)_{2}\left(\left(x_{i}\right)_{1}+\left(x_{i}\right)_{2}\right)} S^{-1}\left(c_{k}\right) S^{-1}\left(b_{j}^{\prime}\right) a_{j}^{(2)} d_{k}^{(2)}(\lambda) .
\end{gathered}
$$

Proof. By Lemma 2.4, we have

$$
\left(q^{2 \lambda}\right)_{1} \mathcal{J}^{1,23}(\lambda)\left(q^{-2 \lambda}\right)_{1}=\left(\mathcal{R}^{23,1}\right)^{-1} \mathcal{J}^{1,23}(\lambda) q^{\sum\left(x_{i}\right)_{1}\left(\left(x_{i}\right)_{2}+\left(x_{i}\right)_{3}\right)} .
$$

Applying $m_{13}\left(S^{-1} \otimes 1 \otimes 1\right)$ to both sides, we get the lemma. 
Now we will use the relations

$$
\begin{gathered}
\sum a_{i}^{\prime(1)} \otimes S^{-1}\left(b_{i}^{\prime}\right) a_{i}^{\prime(2)}=\sum a_{k} \otimes u b_{k}, \\
\mathcal{R}^{23} \mathcal{J}^{1,23}=\mathcal{J}^{1,32} \mathcal{R}^{23}
\end{gathered}
$$

and

$$
\sum S\left(c_{i}\right) d_{i}^{(1)}(\lambda) \otimes d_{i}^{(2)}(\lambda)=S(\mathbb{Q})\left(\lambda-\frac{1}{2} h^{(2)}\right)_{1} \mathcal{J}^{-1}\left(\lambda+\frac{1}{2} \hat{h}^{(1)}\right)
$$

The first relation follows from the coproduct rule for the R-matrix, the second one is the functoriality of $\mathcal{J}$, and the third one is obtained if one applies $m_{12}(S \otimes 1 \otimes 1)$ to the cocycle relation $(2.12)$.

Using (2.29)-(2.31), and the fact that $(1 \otimes S)\left(\mathcal{R}^{-1}\right)=\mathcal{R}$, we get from $(2.27)$

$$
\begin{gathered}
\sum d_{i}^{(1)}(\lambda) \otimes q^{2 \lambda} S^{-1}\left(c_{i}\right) q^{-2 \lambda} d_{i}^{(2)}(\lambda)= \\
\sum\left(d_{k}^{(2)}(\lambda) a_{j}\right)_{1}\left(u^{-1}\right)_{2} q^{-\sum\left(x_{i}\right)_{2}\left(\left(x_{i}\right)_{1}+\left(x_{i}\right)_{2}\right)}\left(S\left(c_{k}\right) d_{k}^{(1)}(\lambda) b_{j}\right)_{2}= \\
q^{-\sum\left(x_{i}\right)_{2}\left(\left(x_{i}\right)_{1}+\left(x_{i}\right)_{2}\right)}\left(u^{-1}\right)_{2} S(\mathbb{Q})\left(\lambda-\frac{1}{2} h\right)_{2}\left(\mathcal{J}^{21}\right)^{-1}\left(\lambda+\frac{1}{2} \hat{h}^{(2)}\right) \mathcal{R}
\end{gathered}
$$

where $\hat{h}^{(2)}$ should be replaced by the weight of the second component of the tensor product after the action of $\mathcal{J}^{-1}$ (i.e.

$$
\mathcal{J}^{-1}\left(\lambda+\frac{1}{2} \hat{h}^{(2)}\right)(v \otimes w)=\sum_{\nu} \mathcal{J}_{\nu}^{-1}\left(\lambda+\frac{1}{2}(w t(w)+\nu)\right)(v \otimes w),
$$

where $w t(w)$ is the weight of $w$, and $\mathcal{J}_{\nu}^{-1}$ is the part of $\mathcal{J}^{-1}$ which shifts the weight of the second component by $\nu$ ).

Substituting (2.32) into (2.26), and using the identity $u^{-1} S(u)=q^{-4 \rho}$, we obtain

$$
\mathcal{M}_{W} \Psi_{V}(\lambda, \mu)=\left.\sum_{\nu} \operatorname{Tr}\right|_{W[\nu]}\left(\tilde{G}(\lambda) \mathbb{R}_{W V}(\lambda)\right) \Psi_{V}(\lambda+\nu, \mu),
$$

where

$$
\tilde{G}(\lambda)=q^{-2 \rho} P(\lambda) S(\mathbb{Q})(\lambda) .
$$

Thus Theorem 2.3 follows from the following Lemma.

Lemma 2.12. $P(\lambda)=\mathbb{Q}^{-1}(\lambda+h)$. Hence $\tilde{G}(\lambda)=G(\lambda+h)$.

Proof. The Lemma is obtained by applying $m_{321}\left(S^{-1} \otimes 1 \otimes S^{-1}\right)$ to the cocycle identity for $\mathbb{J}$.

Theorem 2.3 is proved.

\subsection{Calculation of $G(\lambda)$.}


Proposition 2.13. Let $G(\lambda)=q^{-2 \rho} \mathbb{Q}^{-1}(\lambda) S(\mathbb{Q})(\lambda-h)$. Then

$$
G(\lambda)=\frac{\delta_{q}(\lambda)}{\delta_{q}(\lambda-h)}
$$

The rest of the subsection is the proof of Proposition 2.13 .

\section{Lemma 2.14 .}

$$
\Delta(G(\lambda))=\mathbb{J}(\lambda)\left(G\left(\lambda+h^{(2)}\right) \otimes G(\lambda)\right) \mathbb{J}^{-1}(\lambda) .
$$

Proof. The element $S(\mathbb{Q})(\lambda)$ coincides with $K^{\prime}(-\lambda-\rho)$, where the element $K^{\prime}(\lambda)$ defined in Section 4.2 of [EV]. By Lemma 27 of [EV], one has $\left\langle w, K^{\prime}(\lambda) w^{*}\right\rangle=$ $B_{\lambda, W}\left(w, w^{*}\right)$, where the bilinear form $B_{\lambda, W}: W \otimes{ }^{*} W \rightarrow \mathbb{C}$ of weight zero is defined by the property $(1 \otimes(),) \circ \Phi_{\lambda-\lambda_{w^{*}}}^{w} \Phi_{\lambda}^{w^{*}}=B_{\lambda, W}\left(w, w^{*}\right) I d_{M_{\lambda}}$. Recall the functoriality property of $B_{\lambda, W}$ :

$$
B_{U \otimes W} \circ\left(J_{U W} \otimes J_{*}{ }^{*} U\right)=B_{U} \circ B_{W} .
$$

(for suitable $\lambda$-parameters, which are omitted for brevity). This property implies that

$$
\Delta(\mathbb{Q}(\lambda))=(S \otimes S)\left(\mathbb{J}^{21}(\lambda)^{-1}\right)\left(\mathbb{Q}(\lambda) \otimes \mathbb{Q}\left(\lambda-h^{(1)}\right)\right) \mathbb{J}\left(\lambda-h^{(1)}-h^{(2)}\right)^{-1},
$$

which implies the lemma.

Set $q=e^{\gamma}$, Then $J\left(\frac{\lambda}{\gamma}\right)$ is a power series in $\gamma$ of the form $1+O(\gamma)$, whose terms lie in $U_{q}(\mathfrak{g}) \otimes U_{q}(\mathfrak{g}) \otimes F$, where $F$ is the field of trigonometric functions.

Lemma 2.15. Let $X(\lambda, \gamma)=\sum_{m=0}^{\infty} X_{m}(\lambda) \gamma^{m}$ be a power series of weight zero satisfying the equation

$$
\Delta(X(\lambda, \gamma))=\mathbb{J}(\lambda / \gamma)\left(X\left(\lambda+\gamma h^{(1)}, \gamma\right) \otimes X(\lambda, \gamma)\right) \mathbb{J}^{-1}(\lambda / \gamma),
$$

where $X_{i} \in U_{q}(\mathfrak{g}) \otimes F$, such that $X_{0}(\lambda)=1$. Then:

(i) If $X=1$ on highest weight vectors of all finite dimensional representations, then $X=1$.

(ii) In general, $X=\frac{f(\lambda, \gamma)}{f(\lambda-\gamma h, \gamma)}$, where $f=1+\gamma f_{1}+\gamma^{2} f_{2}+. ., f_{i} \in F$.

Proof. (i) $X_{1}$ is a primitive element, so it lies in $\mathfrak{h} \otimes F$. Since it acts trivially on all highest weight vectors, $X_{1}=0$. Similarly, $X_{i}=0$ for $i>1$ by induction, using the same argument.

(ii) Let $\omega_{i}$ be fundamental weights, and $\eta_{i}(\lambda)$ be the eigenvalues of $X(\lambda)$ on the highest weight vector of the representation with highest weight $\omega_{i}$. It is easy to see that $\eta_{i}\left(\lambda+\gamma \omega_{j}\right) \eta_{j}(\lambda)=\eta_{j}\left(\lambda+\gamma \omega_{i}\right) \eta_{i}(\lambda)$, which implies that there exists $g$ such that $g\left(\lambda+\gamma \omega_{i}\right)=\eta_{i}(\lambda) g(\lambda)$. Let $\tilde{X}(\lambda)=X(\lambda) g(\lambda) / g(\lambda+\gamma h)$. Then $\tilde{X}$ satisfies the conditions of (i), so by (i) $\tilde{X}=1$. This proves (ii) (we can set $f(\lambda)=g(\lambda+\gamma h)$ ). 
Corollary 2.16. $G(\lambda)=\frac{f(\lambda)}{f(\lambda-h)}$ for a suitable function $f$.

Proof. Follows from Lemmas 2.14 and 2.15.

To conclude the proof of Proposition 2.13, it remains to show that in Corollary 2.16 , one can take $f(\lambda)=\delta_{q}(\lambda)$.

To do this, we apply Theorem 2.3 and part (ii) of Proposition 2.2 in the case $V=\mathbb{C}$. In this case $\Psi_{V}(\lambda, \mu)=\frac{q^{2(\mu+\rho, \lambda)}}{\delta_{q}(\lambda)}, R_{W V}=1$. So, using Corollary 2.16, we obtain

$$
\left.\sum_{\nu} \frac{f(\lambda+\nu)}{f(\lambda)}\right|_{W[\nu]} \operatorname{dim} W[\nu] \frac{q^{2(\mu+\rho, \lambda+\nu)}}{\delta_{q}(\lambda+\nu)}=\chi_{W}\left(q^{2(\mu+\rho)}\right) \frac{q^{2(\mu+\rho, \lambda)}}{\delta_{q}(\lambda)}
$$

This equation is obviously satisfied if $f=\delta$. Since the validity of this equation for all $\mu$ completely determines $f(\lambda+h) / f(\lambda)$, we get the lemma.

\subsection{A modification of Theorem 2.3.}

Let $\varphi_{V}(\lambda, \mu)=\Psi_{V}(\lambda, \mu) \delta_{q}(\lambda)$.

\section{Theorem 2.17.}

$$
\mathcal{D}_{W}^{\lambda} \varphi_{V}(\lambda, \mu)=\chi_{W}\left(q^{2(\mu+\rho)}\right) \varphi_{V}(\lambda, \mu) .
$$

Proof. Follows from Theorem 2.3, Proposition 2.2, and Proposition 2.13.

\subsection{Proof of Theorem 1.1.}

The theorem follows from Theorem 2.17 and the fusion identity

$$
\mathbb{J}^{1 \ldots N}(\lambda)^{-1} \mathbb{R}^{0,1 \ldots N}(\lambda) \mathbb{J}^{1 \ldots N}\left(\lambda+h^{(0)}\right)=\mathbb{R}^{01}\left(\lambda+h^{(2 \ldots N)}\right) \ldots \mathbb{R}^{0 N}(\lambda),
$$

which is easily checked from the definition.

\section{The Dual MR equations.}

\subsection{The map $L_{W V^{*}}(\mu)$.}

Let $W$ be a finite dimensional representation of $U_{q}(\mathfrak{g})$. Denote by $H_{\mu W}^{\lambda}$ the isotypic component of type $M_{\lambda}$ in $M_{\mu} \otimes W$. For generic $\mu$, we have a natural isomorphism $\eta: W[\lambda-\mu] \otimes M_{\lambda} \rightarrow H_{\mu W}^{\lambda}$ given by $w \otimes v \rightarrow \Phi_{\lambda}^{w} v$.

Consider the intertwining operator

$$
P_{V \otimes V^{*}, W} \mathcal{R}_{V W}\left(\Phi_{\mu}^{V} \otimes I d_{W}\right): M_{\mu} \otimes W \rightarrow M_{\mu} \otimes W \otimes V \otimes V^{*}
$$

Restricting this operator to the isotypic component of $M_{\mu+\nu}$, from the intertwining property we obtain

$$
\left.P_{V \otimes V^{*}, W} \mathcal{R}_{V W}\left(\Phi_{\mu}^{V} \otimes I d_{W}\right)\right|_{W[\nu] \otimes M_{\mu+\nu}}=L_{W V^{*}}(\mu)\left(I d_{W} \otimes \Phi_{\mu+\nu}^{V}\right),
$$

where $L_{W V^{*}}(\mu): W \otimes V^{*} \rightarrow W \otimes V^{*}$ is a uniquely determined linear map. Our task now is to find this linear map explicitly.

\subsection{Computation of $L_{W V^{*}}$.}




\section{Proposition 3.1.}

$$
L_{W V^{*}}(\mu)=R_{W V}(\mu+\nu)^{t_{2}},
$$

where $t_{2}$ means transposition in the third component.

Proof. Let us apply both sides of (3.2) to $w \otimes y$. Let $B$ be a homogeneous basis of $V$. Then we get

$$
\sum_{v \in B}\left[\eta^{-1} P_{V W} \mathcal{R}_{V W}\left(\Phi_{\mu}^{v} \otimes 1\right) \Phi_{\mu+\nu}^{w} y\right] \otimes v^{*}=\sum_{v \in B} L_{W V^{*}}(\mu) \Phi_{\mu+\nu}^{v}\left(w \otimes y \otimes v^{*}\right) .
$$

Moving $\eta^{-1}$ and $\mathcal{R}$ to the right, and using the notation $L_{W V^{*}}=\sum p_{i} \otimes q_{i}$, we get

$$
\sum_{v \in B}\left[\left(\Phi_{\mu}^{v} \otimes 1\right) \Phi_{\mu+\nu}^{w} y\right] \otimes v^{*}=P_{V W}\left(\mathcal{R}_{V W}^{21}\right)^{-1} \sum_{i} \sum_{v \in B} \Phi_{\mu+\nu-w t(v)}^{p_{i} w} \Phi_{\mu+\nu}^{v} y \otimes q_{i} v^{*}
$$

where $w t(v)$ denotes the weight of $v$.

Simplifying (3.5), we get

$$
\sum_{v \in B} \Phi_{\mu+\nu}^{J(\mu+\nu)(v \otimes w)} y \otimes v^{*}=\sum_{i} \sum_{v \in B} \Phi_{\mu+\nu}^{P_{V W}\left(\mathcal{R}^{21}\right)^{-1} J(\mu+\nu)\left(p_{i} w \otimes q_{i}^{*} v\right)} y \otimes v^{*} .
$$

This implies that on $W[\nu] \otimes V$, one has

$$
J(\mu+\nu)=\mathcal{R}^{-1} J^{21}(\mu+\nu)\left(L_{W V^{*}}^{21}(\mu)\right)^{t_{1}} .
$$

That is,

$$
L_{W V^{*}}(\mu)=\left(J^{-1}(\mu+\nu) \mathcal{R}^{21} J^{21}(\mu+\nu)\right)^{t_{2}}=R_{W V}(\mu+\nu)^{t_{2}} .
$$

\subsection{Proof of Theorem 1.2 .}

Let us multiply both sides of (3.2) on the right by $q^{2 \lambda}$ (acting in $M_{\mu} \otimes W$ ), on the left by $q^{-\left.\left.\sum x_{i}\right|_{V} x_{i}\right|_{W}}$, and compute the trace in the Verma modules, summing over all $\nu$. After multiplication by $\delta_{q}(\lambda)$ and using Proposition 3.1 and the fact that $\varphi_{V}$ has zero weight in $V$, we obtain

$$
\chi_{W}\left(q^{2 \lambda}\right) \varphi_{V}(\lambda, \mu)=\left.\sum_{\nu} \operatorname{Tr}\right|_{W(\nu)}\left(R_{W V}(\mu+\nu)^{t_{2}}\right) \varphi_{V}(\lambda, \mu+\nu) .
$$

This is equivalent to

$$
\chi_{W^{*}}\left(q^{-2 \lambda}\right) \varphi_{V}(\lambda, \mu)=\left.\sum_{\nu} \operatorname{Tr}\right|_{W^{*}(-\nu)}\left(R_{W V}(\mu+\nu)^{t_{1} t_{2}}\right) \varphi_{V}(\lambda, \mu+\nu) .
$$

Rewriting the last equation in terms of $F_{V}(\lambda, \mu)$, we obtain

$\chi_{W^{*}}\left(q^{-2 \lambda}\right) F_{V}(\lambda, \mu)=\left.\left.\left.\sum_{\nu} \operatorname{Tr}\right|_{W^{*}(\nu)} \mathbb{Q}^{-1}(\mu)\right|_{V^{*}}\left(\mathbb{R}_{W V}(\mu+\nu)^{t_{1} t_{2}}\right) \mathbb{Q}(\mu+\nu)\right|_{V^{*}} F_{V}(\lambda, \mu+\nu)$.

The expression $\mathbb{R}_{W V}(\mu)^{t_{1} t_{2}}$ can be computed from (2.38):

$(3.12)$

$\mathbb{R}_{W V}(\mu)^{t_{1} t_{2}}=\left(\mathbb{Q}(\mu) \otimes \mathbb{Q}\left(\mu-h^{(1)}\right)\right) \mathbb{R}_{W^{*} V^{*}}\left(\mu-h^{(1)}-h^{(2)}\right)\left(\mathbb{Q}^{-1}\left(\mu-h^{(2)}\right) \otimes \mathbb{Q}^{-1}(\mu)\right)$.

This expression and (3.11) imply Theorem 1.2. 


\section{The DUAL QKZB EQUATIONS}

In this section, we will prove Theorem 1.4.

Using formula (3) in [EV], we get

$$
\left(\Phi_{\mu-\mu_{v}}^{w} \otimes 1\right) \Phi_{\mu}^{v}=\left(1 \otimes \mathcal{R}_{W V}^{-1} P_{V W}\right) \sum_{k}\left(\Phi_{\mu-\mu_{w_{k}}}^{v_{k}} \otimes 1\right) \Phi_{\mu}^{w_{k}}
$$

where

$$
\sum v_{k} \otimes w_{k}=R_{V W}(\mu)(v \otimes w)
$$

Therefore, using simplified notations, we have

$$
\Phi_{\mu+h^{\left(V^{*}\right)}}^{W} \Phi_{\mu}^{V}=\mathcal{R}^{-1} R_{21}^{*}(\mu) \Phi_{\mu+h^{\left(W^{*}\right)}}^{V} \Phi_{\mu}^{W}=\mathcal{R}_{21} R^{*}(\mu)^{-1} \Phi_{\mu+h^{\left(W^{*}\right)}}^{V} \Phi_{\mu}^{W}
$$

Let us now take the j-th intertwining operator in (1.3) and move it to the right, permuting it with other operators. We have

$$
\begin{gathered}
\Psi_{V_{1} \ldots V_{N}}(\lambda, \mu)=\mathcal{R}_{j+1 j} \ldots \mathcal{R}_{N j}\left(q^{2 \lambda}\right)_{j} R_{j j+1}^{*}\left(\mu+\sum_{i=j+2}^{N} h^{(* i)}\right)^{-1} \ldots R_{j N}^{*}(\mu)^{-1} \times \\
\operatorname{Tr}\left(\Phi_{\mu+\sum_{i=2}^{N} h^{(* i)}}^{V_{1}} \ldots \Phi_{\mu+\sum_{i=j}^{N} h^{(* i)}}^{V_{j-1}} \Phi_{\mu+h^{(* j)}+\sum_{i=j+2}^{N} h^{(* i)}}^{V_{j+1}} \ldots \Phi_{\mu+h^{(* j)}}^{V_{N}} q^{2 \lambda} \Phi_{\mu}^{V_{j}}\right)= \\
\mathcal{R}_{j+1 j} \ldots \mathcal{R}_{N j}\left(q^{2 \lambda}\right)_{j} R_{j j+1}^{*}\left(\mu+\sum_{i=j+2}^{N} h^{(* i)}\right)^{-1} \ldots R_{j N}^{*}(\mu)^{-1} \Gamma_{* j} \times
\end{gathered}
$$

$$
\operatorname{Tr}\left(\Phi_{\mu+\sum_{i=2, i \neq j}^{V_{1}} h^{(* i)}}^{N} \ldots \Phi_{\mu+\sum_{i=j+1}^{N} h^{(* i)}}^{V_{j-1}} \Phi_{\mu+\sum_{i=j+2}^{N} h^{(* i)}}^{V_{j+1}} \ldots \Phi_{\mu}^{V_{N}} q^{2 \lambda} \Phi_{\mu+\sum_{i \neq j} h^{(* i)}}^{V_{j}}\right)
$$

(in the last equality we use that $\sum h^{(* i)}=0$ ). Using the cyclic property of the trace, we can now put the $\mathrm{j}$-th operator into the beginning, and move it to the right to its original place, thus completing the cycle. This yields

$$
\begin{gathered}
\Psi_{V_{1} \ldots V_{N}}(\lambda, \mu)=\mathcal{R}_{j+1 j} \ldots \mathcal{R}_{N j}\left(q^{2 \lambda}\right)_{j} \mathcal{R}_{j 1}^{-1} \ldots \mathcal{R}_{j j-1}^{-1} \times \\
R_{j j+1}^{*}\left(\mu+\sum_{i=j+2}^{N} h^{(* i)}\right)^{-1} \ldots R_{j N}^{*}(\mu)^{-1} \Gamma_{* j} R_{1 j}^{*}\left(\mu+\sum_{i=2, i \neq j}^{N} h^{(* i)}\right) \ldots R_{j-1 j}^{*}\left(\mu+\sum_{i=j+1}^{N} h^{(* i)}\right) \times \\
\Psi_{V_{1} \ldots V_{N}}(\lambda, \mu) .
\end{gathered}
$$

Multiplying both sides of (4.5) by $\delta_{q}(\lambda)$, replacing $\mu$ by $-\mu-\rho$, and multiplying by $\mathbb{J}^{1 \ldots N}(\lambda)^{-1}$ and the product of $\mathbb{Q}^{-1}$-s, we obtain

$$
\begin{gathered}
F_{V_{1} \ldots V_{N}}(\lambda, \mu)=\mathbb{J}^{1 \ldots N}(\lambda)^{-1} \mathcal{R}_{j+1 j} \ldots \mathcal{R}_{N j}\left(q^{2 \lambda}\right)_{j} \mathcal{R}_{j 1}^{-1} \ldots \mathcal{R}_{j j-1}^{-1} \mathbb{J}^{1 \ldots N}(\lambda) \\
{\left[\mathbb{Q}^{-1}(\mu)^{(* N)} \otimes \ldots \otimes \mathbb{Q}^{-1}\left(\mu-h^{(* 2 \ldots * N)}\right)^{(* 1)}\right] \times} \\
\mathbb{R}_{j j+1}^{*}\left(\mu-\sum_{i=j+2}^{N} h^{(* i)}\right)^{-1} \ldots \mathbb{R}_{j N}^{*}(\mu)^{-1} \Gamma_{* j}^{-1} \mathbb{R}_{1 j}^{*}\left(\mu-\sum_{i=2, i \neq j}^{N} h^{(* i)}\right) \ldots \mathbb{R}_{j-1 j}^{*}\left(\mu-\sum_{i=j+1}^{N} h^{(* i)}\right) \times \\
{\left[\left(\mathbb{Q}(\mu)^{(* N)} \otimes \ldots \otimes \mathbb{Q}\left(\mu-h^{(* 2 \ldots * N)}\right)^{(* 1)}\right] F_{V_{1} \ldots V_{N}}(\lambda, \mu) .\right.}
\end{gathered}
$$


Inverting (4.6), we get

$$
\begin{gathered}
F_{V_{1} \ldots V_{N}}(\lambda, \mu)=\mathbb{J}^{1 \ldots N}(\lambda)^{-1} \mathcal{R}_{j j-1} \ldots \mathcal{R}_{j 1}\left(q^{-2 \lambda}\right)_{j} \mathcal{R}_{N j}^{-1} \ldots \mathcal{R}_{j+1 j}^{-1} \mathbb{J}^{1 \ldots N}(\lambda) \\
{\left[\mathbb{Q}^{-1}(\mu)^{(* N)} \otimes \ldots \otimes \mathbb{Q}^{-1}\left(\mu-h^{(* 2 \ldots * N)}\right)^{(* 1)}\right] \times} \\
\mathbb{R}_{j-1 j}^{*}\left(\mu-\sum_{i=j+1}^{N} h^{(* i)}\right)^{-1} \ldots \mathbb{R}_{1 j}^{*}\left(\mu-\sum_{i=2, i \neq j}^{N} h^{(* i)}\right)^{-1} \Gamma_{* j} \mathbb{R}_{j N}^{*}(\mu) \ldots \mathbb{R}_{j j+1}^{*}\left(\mu-\sum_{i=j+2}^{N} h^{(* i)}\right) \times \\
{\left[(4.7) \quad\left[(\mu)^{(* N)} \otimes \ldots \otimes \mathbb{Q}\left(\mu-h^{(* 2 \ldots * N)}\right)^{(* 1)}\right] F_{V_{1} \ldots V_{N}}(\lambda, \mu) .\right.}
\end{gathered}
$$

Using formula (3.12), it is not difficult to check that on zero weight vectors

$$
\begin{gathered}
{\left[\mathbb{Q}^{-1}(\mu)^{(* N)} \otimes \ldots \otimes \mathbb{Q}^{-1}\left(\mu-h^{(* 2 \ldots * N)}\right)^{(* 1)}\right] \times} \\
\mathbb{R}_{j-1 j}^{*}\left(\mu-\sum_{i=j+1}^{N} h^{(* i)}\right)^{-1} \ldots \mathbb{R}_{1 j}^{*}\left(\mu-\sum_{i=2, i \neq j}^{N} h^{(* i)}\right)^{-1} \Gamma_{* j} \mathbb{R}_{j N}^{*}(\mu) \ldots \mathbb{R}_{j j+1}^{*}\left(\mu-\sum_{i=j+2}^{N} h^{(* i)}\right) \times \\
(4.8) \quad\left[\mathbb{Q}(\mu)^{(* N)} \otimes \ldots \otimes \mathbb{Q}\left(\mu-h^{(* 2 \ldots * N)}\right)^{(* 1)}\right]=K_{j}^{\vee} .
\end{gathered}
$$

So it remains to check that

$$
\mathbb{J}^{1 \ldots N}(\lambda)^{-1} \mathcal{R}_{j j-1} \ldots \mathcal{R}_{j 1}\left(q^{-2 \lambda}\right)_{j} \mathcal{R}_{N j}^{-1} \ldots \mathcal{R}_{j+1 j}^{-1} \mathbb{J}^{1 \ldots N}(\lambda)=D_{j}^{\vee}
$$

(on zero weight vectors).

To check (4.9), observe that it can be written in the form

$$
\mathbb{J}^{1 \ldots N}(\lambda)^{-1} \mathcal{R}_{j, 1 \ldots j-1}\left(q^{-2 \lambda}\right)_{j} \mathcal{R}_{j+1 \ldots N, j^{-1}}^{-1 \ldots N}(\lambda)=D_{j}^{\vee},
$$

which implies that it is enough to check (4.9) for $N=2$ and $N=3$.

Let $E_{j}$ be the left hand side of (4.9). Since $E_{1} E_{2} E_{3}=D_{1}^{\vee} D_{2}^{\vee} D_{3}^{\vee}=1$ for $N=3$, it is enough to prove (4.9) for $N=2$ and $N=3, j=1,3$. But the last two cases follow from the case $N=2$, so it is enough to check only this case.

If $N=2$, identity (4.9) easily follows from Lemma 2.4. Theorem 1.4 is proved.

\section{The SYMmetRY IDENTITY.}

In this section we will prove Theorem 1.5 using Theorems 1.1 and 1.2.

Let $V$ be a finite dimensional representation of $U_{q}(\mathfrak{g})$, and $v \in V$ a homogeneous vector. Consider the operator $\Phi_{\mu}^{v}$ as a linear operator $U_{q}\left(\mathfrak{n}_{-}\right) \rightarrow U_{q}\left(\mathfrak{n}_{-}\right) \otimes V$, identifying the Verma modules with $U_{q}\left(\mathfrak{n}_{-}\right)$.

Lemma 5.1. The operator $\Phi_{\mu}^{v}$ is a rational function of variables $q^{2\left(\mu, \alpha_{i}\right)}$ of the form $\tilde{\Phi}_{\mu}^{v} / D(\mu)$, where $\tilde{\Phi}_{\mu}^{V}$ is an operator valued polynomial in $x_{i}:=q^{2\left(\mu, \alpha_{i}\right)}$, and $D(\mu)$ is a polynomial in $x_{i}$ with nonzero free term.

Proof. This follows from the arguments in the proof of Proposition 2.2 in [ES].

Corollary 5.2. The matrix elements of the universal trace function $\Psi$ can be expanded in formal series belonging to the space $q^{2(\lambda, \mu)} \mathbb{C}\left[\left[q^{-2\left(\lambda, \alpha_{i}\right)}\right]\right] \otimes \mathbb{C}\left[\left[q^{2\left(\mu, \alpha_{i}\right)}\right]\right]$.

Remark. Note that the tensor product in Corollary 5.2 is algebraic, i.e. uncompleted.

Let $K=q^{-2(\lambda, \mu)} \mathbb{C}\left[\left[q^{-2\left(\lambda, \alpha_{i}\right)}\right]\right] \otimes \mathbb{C}\left[\left[q^{-2\left(\mu, \alpha_{i}\right)}\right]\right] . \quad$ Denote by $L$ the space $\left(V_{1} \otimes \ldots \otimes V_{N}\right)[0] \otimes K$. 
Lemma 5.3. For any vector $v \in V_{1} \otimes \ldots \otimes V_{N}[0]$, the function $\left\langle F_{V_{1} \ldots V_{n}}, v\right\rangle$ belongs to $L$.

Proof. It is clear from Proposition 2.2 of $[\mathrm{ES}]$ and the definition of $\mathbb{J}$ that for any $V, W$ the function $\mathbb{J}_{V W}(\lambda)$ is a rational function of $y_{i}=q^{-2\left(\lambda, \alpha_{i}\right)}$, which can be represented as a ratio of an operator-valued polynomial of $y_{i}$ whose free term is invertible, and a scalar polynomial of $y_{i}$ with nonzero free term. The same statement, for the same reason, applies to $\mathbb{Q}(\lambda)$. These facts and Corollary 5.2 imply the Lemma.

Let $L_{0} \subset L$ be the space of solutions of equations (1.11) whose highest term (as of a series in $\left.x_{i}=q^{-2\left(\lambda, \alpha_{i}\right)}\right)$ is $v q^{-2(\lambda, \mu)}$, where $v \in\left(V_{1} \otimes \ldots V_{N}\right)[0]$ is independent of $\mu$. By Theorem 1.1 and Lemma 5.3, for any $v \in\left(V_{1} \otimes \ldots \otimes V_{N}\right)[0]$ the inner product $\left\langle F_{V_{1}, \ldots, V_{N}}, v\right\rangle$ is in $L_{0}$.

Lemma 5.4. $L_{0}$ is a complex vector space of dimension $d=\operatorname{dim}\left(V_{1} \otimes \ldots \otimes V_{N}\right)[0]$. Moreover, if $B$ is a basis of $\left(V_{1} \otimes \ldots \otimes V_{N}\right)[0]$ then the collection of functions $\left\langle F_{V_{1}, \ldots, V_{N}}, v\right\rangle, v \in B$, is a basis of $L_{0}$.

Proof. The solutions $\left\langle F_{V_{1}, \ldots, V_{N}}, v\right\rangle, v \in B$ are linearly independent, since, by the arguments in the proof of Lemma 5.3, so are their highest terms. So it remains to show that the dimension of the space $L_{0}$ is not bigger than $d$.

Let $I$ be the maximal ideal in $\mathbb{C}\left[\left[x_{1}, \ldots, x_{r}\right]\right]$. It is clear that for any $m \geq 1$ the operator $\mathcal{D}_{W}-\chi_{W}\left(q^{-2 \mu}\right)$ preserves the subspace $I^{m} L$, so it is enough to check that the dimension of the space of solutions is at most $d$ on $L / I^{m} L$ for all $m$.

For $s \in \mathbb{C}^{*}$, define an automorphism $g_{s}$ of $L$ by $g_{s} f\left(x_{1}, \ldots, x_{r}\right)=f\left(s x_{1}, \ldots, s x_{r}\right)$. Let $\mathcal{D}_{W}^{0}=\lim _{s \rightarrow 0} g_{s} \mathcal{D}_{W} g_{s}^{-1}$. It is enough to show that the system $\mathcal{D}_{W}^{0} f=$ $\chi_{W}\left(q^{-2 \mu}\right) f$ has no more than $d$ linearly independent solutions.

The operator $D_{W}^{0}$ is easily computed. Namely, since the exchange matrices converge to the usual R-matrices as $\lambda \rightarrow \infty$ (see Theorem 50 of [EV]), one gets

$$
\mathcal{D}_{W}^{0}=\sum_{\nu} \operatorname{dim}(W[\nu]) T_{\nu}
$$

Therefore, the operators $\mathcal{D}_{W}^{0}$ are diagonal in the monomial basis, and it is obvious that the system $\mathcal{D}_{W}^{0} f=\chi_{W}\left(q^{-2 \mu}\right) f$ has no more than $d$ linearly independent solutions.

Corollary 5.5. There exists a unique element $M(\mu) \in \operatorname{End}\left(\left(V_{N}^{*} \otimes \ldots \otimes V_{1}^{*}\right)[0]\right) \otimes$ $\mathbb{C}\left[\left[q^{-2\left(\mu, \alpha_{i}\right)}\right]\right]$ such that

$$
F_{V_{N}^{*}, \ldots, V_{1}^{*}}^{*}(\mu, \lambda)=(1 \otimes M(\mu)) F_{V_{1}, \ldots, V_{N}}(\lambda, \mu) .
$$

Proof. This follows from Lemma 5.4 and Theorem 1.2.

Now we will prove Theorem 1.5. Applying Corollary 5.5 twice, we obtain that $M(\mu) M^{\prime}(\lambda)=1$ for some function $M^{\prime}(\lambda)$. This implies that $M$ is in fact independent of $\mu$. Looking at the highest terms of $F$ and $F^{*}$, one finds that $M=1$.

This proves Theorem 1.5. 


\section{THE SYMMETRY OF THE UNIVERSAL TRACE \\ FUNCTION UNDER $q \rightarrow q^{-1}$, AND THE FUNCTION $u_{V}$.}

\subsection{The symmetry identity with $q \rightarrow q^{-1}$.}

In this section we will study the behavior of the functions $\Psi_{V}$ under the transformation $q \rightarrow q^{-1}$.

Consider the algebra isomorphism $\xi: U_{q}(\mathfrak{g}) \rightarrow U_{q^{-1}}(\mathfrak{g})$ given by $\xi\left(E_{i}\right)=E_{i} q^{d_{i} h_{i}}$, $\xi\left(F_{i}\right)=q^{-d_{i} h_{i}} F_{i}, \xi\left(q^{h}\right)=q^{-h}$. Using this isomorphism, we will identify $U_{q}(\mathfrak{g})$ and $U_{q^{-1}}(\mathfrak{g})$ (as algebras), and regard any module over $U_{q}(\mathfrak{g})$ as a module over $U_{q^{-1}}(\mathfrak{g})$ and vice versa.

We will start by proving the following theorem.

Theorem 6.1. The function $\Psi_{V_{1}, . ., V_{N}}$ satisfies the equality

$$
\Psi_{V}\left(q^{-1},-\lambda, \mu\right)=u(q)^{-1} \mathbb{Q}(q, \lambda) \Psi_{V}(q, \lambda, \mu),
$$

where $u(q)$ denotes the Drinfeld element $u$ of $U_{q}(\mathfrak{g})$.

Remark. To avoid confusion, here and below we explicitly specify for every expression whether it is evaluated at $q$ or at $q^{-1}$.

Proof. It is obvious that $\xi$ is an antiisomorphism of coalgebras. This implies that for a finite dimensional representation $V$ and a vector $v \in V[0]$ the operator $P \Phi_{\mu}^{v}(q)$ is an intertwining operator $M_{\mu} \rightarrow V \otimes M_{\mu}$ for $U_{q^{-1}}(\mathfrak{g})$, and hence the operator $\left(\mathcal{R}^{10}\right)^{-1} \Phi_{\mu}^{v}(q)$ is an intertwining operator $M_{\mu} \rightarrow M_{\mu} \otimes V$ for $U_{q^{-1}}(\mathfrak{g})$. We have $\left(\mathcal{R}^{10}\right)^{-1}(q) \Phi_{\mu}^{v}(q) v_{\mu}=v_{\mu} \otimes v+\ldots$, so we have

$$
\left(\mathcal{R}^{10}\right)^{-1}(q) \Phi_{\mu}^{V}(q)=\Phi_{\mu}^{V}\left(q^{-1}\right) .
$$

Let us multiply both sides of (6.2) by $q^{2 \lambda}$ and take the trace.

Using (2.15) and (2.16), we get

$$
\operatorname{Tr}\left(\left(\mathcal{R}^{10}\right)^{-1}(q) \Phi_{\mu}^{V}(q) q^{2 \lambda}\right)=u(q)^{-1} \mathbb{Q}(q, \lambda) \Psi_{V}(q, \lambda, \mu),
$$

which implies the theorem.

\subsection{The function $\hat{u}_{V}(\lambda, \mu)$.}

Now let $V$ be an irreducible $U_{q}(\mathfrak{g})$-module, and define the function

$$
\hat{u}_{V}(\lambda, \mu):=\delta_{q}(\lambda) \Psi_{V}\left(q^{-1},-\lambda,-\mu-\rho\right) .
$$

Corollary 6.2. The function $\hat{u}_{V}$ is symmetric: $\hat{u}_{V}(\lambda, \mu)=\hat{u}_{V^{*}}^{*}(\mu, \lambda)$.

Proof. Let $\nu$ be the highest weight of $V$. By Theorem 6.1, we have

$\hat{u}_{V}(\lambda, \mu)=q^{-(\nu, \nu+2 \rho)} \delta_{q}(\lambda) \mathbb{Q}(q, \lambda) \Psi_{V}(\lambda,-\mu-\rho)=q^{-(\nu, \nu+2 \rho)}(\mathbb{Q}(q, \lambda) \otimes \mathbb{Q}(q, \mu)) F_{V}(\lambda, \mu)$.

Therefore, the symmetry of $\hat{u}_{V}$ follows from Theorem 1.5. 


\subsection{The function $u_{V}(\lambda, \mu)$.}

The function $\hat{u}_{V}$ has poles. We would like to get rid of them by multiplying the function $\hat{u}_{V}$ by its denominator.

Following [ES], introduce the function

$$
\delta_{V}(\mu)=\prod_{\alpha>0} \prod_{n=1}^{k_{\alpha}^{V}}\left(q^{(\alpha, \mu+\rho)-n(\alpha, \alpha) / 2}-q^{-(\alpha, \mu+\rho)+n(\alpha, \alpha) / 2}\right),
$$

where for a positive root $\alpha$ we define $k_{\alpha}^{V}:=\max \{n: V[n \alpha] \neq 0\}$.

Define the function

$$
u_{V}(\lambda, \mu):=\delta_{V^{*}}(-\lambda-\rho) \delta_{V}(-\mu-\rho) \hat{u}_{V}(\lambda, \mu) .
$$

Proposition 6.3. The function $u_{V}$ is symmetric: $u_{V}(\lambda, \mu)=u_{V^{*}}^{*}(\mu, \lambda)$, and is a product of $q^{-2(\lambda, \mu)}$ and a trigonometric polynomial of $\lambda$ and $\mu$. In particular, it is holomorphic in $\lambda, \mu$.

Proof. The symmetry of $u_{V}$ follows from Corollary 6.2. The fact that $u_{V}$ is holomorphic in $\mu$ is a consequence of Lemma 5.1. The fact that $u_{V}$ is holomorphic in $\lambda$ follows from the symmetry.

Using (6.5), one gets the following expression of $u_{V}$ in terms of $F_{V}$ :

$$
u_{V}(\lambda, \mu)=q^{-(\nu, \nu+2 \rho)}\left(\delta_{V^{*}}(-\lambda-\rho) \mathbb{Q}(q, \lambda) \otimes \delta_{V}(-\mu-\rho) \mathbb{Q}(q, \mu)\right) F_{V}(\lambda, \mu) .
$$

In Section 8 we will show that the function $u_{V}$ for $U_{q}\left(s l_{2}\right)$ coincides (up to a constant factor) with the trigonometric limit of the universal hypergeometric function $u$ introduced in [FV2].

\section{Calculation of the functions $u_{V}$ And $F_{V}$ For $s l_{2}$}

\subsection{Calculation of the trace function.}

Recall that $U_{q}\left(s l_{2}\right)$ is generated by $E, F, q^{h}$, with relations

$$
q^{h} E q^{-h}=q^{2} E, q^{h} F q^{-h}=q^{-2} F, E F-F E=\frac{q^{h}-q^{-h}}{q-q^{-1}},
$$

and the coproduct is defined by

$$
\Delta(E)=E \otimes q^{h}+1 \otimes E, \Delta(F)=F \otimes 1+q^{-h} \otimes F .
$$

Weights for $U_{q}\left(s l_{2}\right)$ can be identified with complex numbers: we say that a vector $v$ in an $U_{q}\left(s l_{2}\right)$-module has weight $\mu$ if $q^{h} v=q^{\mu} v$. In this case we have $\left(\mu, \mu^{\prime}\right)=\mu \mu^{\prime} / 2$. We will write $q^{\lambda}$ for $q^{(\lambda, \alpha)}$. Thus the meaning of $q^{\lambda}$ in this section is different from the previous sections.

Recall also that for any number $a$ the q-number $[a]_{q}$ is defined by $[a]_{q}=\frac{q^{a}-q^{-a}}{q-q^{-1}}$.

Consider the function $\Psi_{V}(\lambda, \mu)$, where $V$ is the representation of $U_{q}\left(s l_{2}\right)$ with highest weight $2 m, m \in \mathbb{Z}_{+}$. Since the weight space $V[0]$ is 1-dimensional, this function can be considered as a scalar function. 
Theorem 7.1. The function $\Psi_{V}(\lambda, \mu)$ is given by the formula

$$
\Psi_{V}(\lambda, \mu)=
$$

$$
q^{\lambda \mu} \sum_{l=0}^{m} q^{l(l-1) / 2}\left(q-q^{-1}\right)^{l} \frac{[m+l]_{q} !}{[l]_{q} ![m-l]_{q} !} \frac{q^{-2 l \lambda}}{\prod_{j=0}^{l-1}\left(1-q^{2(\mu-j)}\right) \prod_{j=0}^{l}\left(1-q^{-2(\lambda-j)}\right)},
$$

where $[n]_{q} !=[1]_{q} \ldots[n]_{q}$.

Proof. We fix a generator $w_{0}$ of $V[0]$. Let us compute the intertwining operator $\Phi_{\mu}: M_{\mu} \rightarrow M_{\mu} \otimes V$.

Let $w_{\beta}, \beta=m, m-1, \ldots,-m$, be the basis of $V$ defined by the condition $F w_{\beta}=$ $w_{\beta-1}$ if $\beta \neq-m$ (this basis is unique up to a common scalar). The image of the highest weight vector under the operator $\Phi_{\mu}$ has the form

$$
\Phi_{\mu} v_{\mu}=\sum_{j=0}^{m} c_{j}(\mu) F^{j} v_{\mu} \otimes w_{j}
$$

where $c_{0}=1$.

Let us compute the coefficients $c_{j}(\mu)$. They are computed from the condition that $\Delta(E)=E \otimes q^{h}+1 \otimes E$ annihilates the r.h.s. of (7.4). Using the formula

$$
e w_{\beta}=[m+\beta+1]_{q}[m-\beta]_{q} w_{\beta+1}, \beta \neq m,
$$

we can rewrite this condition in the form

$$
q^{2 i}[i]_{q}[\mu-i+1]_{q} c_{i}(\mu)+[m+i]_{q}[m-i+1]_{q} c_{i-1}(\mu)=0, i \geq 1 .
$$

Solving this recurrence relation, we obtain

$$
c_{i}(\mu)=(-1)^{i} q^{-i(i+1)} \frac{[m+i]_{q} !}{[i]_{q} ![m-i]_{q} !} \prod_{j=1}^{i}[\mu-i+1]_{q}^{-1} .
$$

Now we need to compute $\Phi_{\mu} F^{k} v_{\mu}$. For this, we need to compute $\Delta\left(F^{k}\right)$. Using the q-binomial theorem, we obtain

$$
\Delta\left(F^{k}\right)=\left(F \otimes 1+q^{-h} \otimes F\right)^{k}=\sum_{l=0}^{k}\left(\begin{array}{l}
k \\
l
\end{array}\right)_{q^{-2}} q^{-l h} F^{k-l} \otimes F^{l},
$$

where

$$
\left(\begin{array}{l}
k \\
l
\end{array}\right)_{p}:=\frac{\prod_{i=k-l+1}^{k}\left(1-p^{i}\right)}{\prod_{i=1}^{l}\left(1-p^{i}\right)} .
$$

Now, using the intertwining property of $\Phi_{\mu}$, we obtain

$$
\begin{gathered}
\Phi_{\mu} F^{k} v_{\mu}=\Delta\left(F^{k}\right) \Phi_{\mu} v_{\mu}= \\
\left(\sum_{l=0}^{k}\left(\begin{array}{l}
k \\
l
\end{array}\right)_{q^{-2}} q^{-l h} F^{k-l} \otimes F^{l}\right) \sum_{j=0}^{m} c_{j}(\mu) F^{j} v_{\mu} \otimes w_{j},
\end{gathered}
$$


This double sum reduces to a single sum if we use the following version of the q-binomial theorem:

$$
\sum_{k \geq l}\left(\begin{array}{l}
k \\
l
\end{array}\right)_{p} x^{k-l}=\prod_{i=0}^{l}\left(1-p^{i} x\right)^{-1} .
$$

Substituting (7.7) into (7.10) and using (7.11), we obtain (7.3). The Theorem is proved.

\section{Corollary 7.2.}

$$
u_{V}(\lambda, \mu)=
$$

$q^{-\lambda \mu} \sum_{l=0}^{m} q^{-l(l-1) / 2}\left(q-q^{-1}\right)^{l} \frac{[m+l]_{q} !}{[l]_{q} ![m-l]_{q} !} q^{-l(\lambda+\mu)} \prod_{j=l+1}^{m}\left(q^{\lambda+j}-q^{-\lambda-j}\right)\left(q^{\mu+j}-q^{-\mu-j}\right)$.

Proof. The statement is obtained from Theorem 7.1 and formulas (6.4) and (6.7).

The function $u_{V}$ is manifestly symmetric in $\lambda$ and $\mu$, as predicted by Proposition 6.3 .

\subsection{A formula for $\mathbb{Q}(\mu)$.}

Now we would like to compute the function $F_{V}$. So it remains to compute the value of $\mathbb{Q}(\mu)$ on the zero-weight subspace of $V$. This value is given by the following Lemma.

Lemma 7.2. The element $\mathbb{Q}(\mu)$ acts on the zero weight subspace of $V$ by the formula

$$
\left.\mathbb{Q}(\mu)\right|_{V[0]}=q^{-2 m} \prod_{j=1}^{m} \frac{q^{-2 \mu-2 j+2}-q^{-2 m}}{q^{-2 \mu-2 j}-1} .
$$

Proof. Denote by $\mathbb{Q}_{r, l}(\mu)$ the eigenvalue of $\mathbb{Q}(\mu)$ on the subspace of weight $l$ of the representation of $U_{q}\left(s l_{2}\right)$ with highest weight $r$.

It is clear that $\mathbb{Q}_{0,0}=1$. The values of $\mathbb{Q}_{1,1}$ and $\mathbb{Q}_{1,-1}$ are easily computed from the definition. Namely, from the ABRR equation we have

$$
\mathcal{J}(\mu)=1+\frac{q^{-1}-q}{q^{-2 \mu}-q^{-2}\left(q^{-h} \otimes q^{h}\right)} F \otimes E+\ldots,
$$

and therefore

$$
\mathbb{Q}_{1,-1}=1, \mathbb{Q}_{1,1}=\frac{q^{-2 \mu}-q^{-2}}{q^{-2 \mu}-1}
$$

Now consider the subspace of weight $l$ in the tensor product $\mathbb{C}^{2} \otimes W$, where $W$ has highest weight $r$. Take the determinant of both sides of (2.38) restricted to this subspace. Using the strict triangularity of $\mathbb{J}$, we can ignore the $\mathbb{J}$ terms and obtain

$$
\mathbb{Q}_{r+1, l}(\mu) \mathbb{Q}_{r-1, l}(\mu)=\mathbb{Q}_{r, l-1}(\mu-1) \mathbb{Q}_{r, l+1}(\mu+1) \frac{q^{-2 \mu}-q^{-2}}{q^{-2 \mu}-1}
$$


This implies

$$
\frac{\mathbb{Q}_{r+1, l}(\mu)}{\mathbb{Q}_{r, l+1}(\mu+1)}=\frac{\mathbb{Q}_{r, l-1}(\mu-1)}{\mathbb{Q}_{r-1, l}(\mu)} \frac{q^{-2 \mu}-q^{-2}}{q^{-2 \mu}-1}
$$

from which we get

$$
\frac{\mathbb{Q}_{r+1, l}(\mu)}{\mathbb{Q}_{r, l+1}(\mu+1)}=\prod_{j=0}^{(r+l-1) / 2} \frac{q^{-2 \mu+2 j}-q^{-2}}{q^{-2 \mu+2 j}-1}=\frac{q^{-2 \mu}-q^{-r-l-1}}{q^{-2 \mu}-1}
$$

This yields

$$
\mathbb{Q}_{r+1, l}(\mu)=\prod_{j=0}^{(r-l+1) / 2} \frac{q^{-2 \mu-2 j}-q^{-r-l-1}}{q^{-2 \mu-2 j}-1}
$$

In particular,

$$
\mathbb{Q}_{2 m, 0}(\mu)=\prod_{j=0}^{m} \frac{q^{-2 \mu-2 j}-q^{-2 m}}{q^{-2 \mu-2 j}-1}=q^{-2 m} \prod_{j=1}^{m} \frac{q^{-2 \mu-2 j+2}-q^{-2 m}}{q^{-2 \mu-2 j}-1},
$$

as desired.

7.3. Calculation of $F_{V}(\lambda, \mu)$.

Proposition 7.3. The function $F_{V}(\lambda, \mu)$ is given by

$$
q^{2 m} \sum_{l=0}^{m} q^{l(l-1) / 2}\left(q-q^{-1}\right)^{l} \frac{[m+l]_{q} !}{[l]_{q} ![m-l]_{q} !} \frac{q^{-2 l \lambda}}{\prod_{j=1}^{l}\left(1-q^{-2(\mu+j)}\right) \prod_{j=1}^{l}\left(1-q^{-2(\lambda-j)}\right)},
$$

$$
F_{V}(\lambda, \mu)=q^{-\lambda \mu} \prod_{j=1}^{m} \frac{q^{-2 \mu-2 j}-1}{q^{-2 \mu-2 j+2}-q^{-2 m}} \times
$$

Proof. Using the definition of $F_{V}$, Theorem 7.1 and Lemma 7.2, we get (7.20).

Remark. Note that expression (7.20) is not manifestly symmetric. In fact, the separate terms in the sum (7.20) are not symmetric, and it is only the whole sum that has the symmetry $\lambda \rightarrow \mu$.

\section{Integral Representation of the trace function $u_{V}$ FOr $\mathfrak{g}=s l_{2}$}

In [FV2], G.Felder and the second author, studying the qKZB difference equations, defined the universal hypergeometric function $u_{m}(\lambda, \tau, \mu, p)$ (depending on a parameter $q$ ) with a number of interesting properties. In this section we will consider the trigonometric limit of this function, and will show that it coincides, up to a constant factor, with the function $u_{V(m)}$ defined by $(6.8)$, where $V(m)$ is the irreducible representation of $U_{q}\left(s l_{2}\right)$ with highest weight $2 \mathrm{~m}$.

By definition, the trigonometric limit of $u_{m}(\lambda, \tau, \mu, p)$ is the leading coefficient of the asymptotic expansion of $u_{m}(\lambda, \tau, \mu, p)$ as the modular parameters $\tau, p$ tend to $i \infty$. We will denote this leading coefficient by $u_{m}(\lambda, \mu)$. 
Sending $\tau, p$ to $i \infty$ in the definition of [FV2], one obtains the following definition of the function $u_{m}(\lambda, \mu)$.

Let $q=e^{t}$ with $\operatorname{Re}(t)>0$, and let $0<|A|<1$. Define a function

$$
\begin{aligned}
& I_{m}(\lambda, \mu, A)=q^{-\lambda \mu} \int_{\left|T_{1}\right|=\ldots=\left|T_{m}\right|=1} \prod_{j=1}^{m} \frac{\left(T_{j} A^{-2} q^{\lambda+m}-q^{-\lambda-m}\right)\left(T_{j} A^{-2} q^{\mu+m}-q^{-\mu-m}\right)}{\left(1-T_{j} A^{2}\right)\left(1-T_{j} A^{-2}\right)} \times \\
& (8.1) \quad \prod_{1 \leq i<j \leq m} \frac{q^{-2}\left(1-T_{i} T_{j}^{-1}\right)^{2}}{\left(1-T_{i} T_{j}^{-1} q^{-2}\right)\left(1-T_{i} T_{j}^{-1} q^{2}\right)} \wedge_{j=1}^{m} \frac{d T_{j}}{2 \pi \sqrt{-1} T_{j}} .
\end{aligned}
$$

It is obvious that this function analytically continues to a rational function in $A$. We will denote this analytic continuation also by $I_{m}$.

\section{Definition.}

$$
u_{m}(\lambda, \mu)=I_{m}\left(\lambda, \mu, q^{m}\right) .
$$

Remark. Note that $A=q^{m}$ does not satisfy the condition $|A|<1$, which is why we needed to talk about the analytic continuation.

The main result of this section is

Theorem 8.1. Let $V(m)$ be the irreducible representation of $U_{q}\left(s l_{2}\right)$ with highest weight $2 m$. Then

$$
u_{V(m)}(\lambda, \mu)=q^{(3 m-1) m} \frac{[2 m]_{q} !}{m !}\left(q-q^{-1}\right)^{m} u_{m}(\lambda, \mu),
$$

where $u_{V(m)}$ is as in Section 6.

The rest of the section is the proof of Theorem 8.1.

\section{Lemma 8.2.}

$\int_{\left|T_{1}\right|=\ldots=\left|T_{m}\right|=1} \prod_{1 \leq i<j \leq m} \frac{\left(1-T_{i} T_{j}^{-1}\right)^{2}}{\left(1-T_{i} T_{j}^{-1} q^{-2}\right)\left(1-T_{i} T_{j}^{-1} q^{2}\right)} \wedge_{j=1}^{m} \frac{d T_{j}}{2 \pi \sqrt{-1} T_{j}}=q^{-m(m-1) / 2} \frac{m !}{[m]_{q} !}$.

Proof. Let $0 \leq|p|<1$. Consider the Macdonald denominator of type $A_{m-1}$ (see [M1])

$$
\Delta_{p, t}\left(x_{1}, \ldots, x_{m}\right)=\prod_{i \neq j} \frac{\left(x_{i} x_{j}^{-1}, p\right)_{\infty}}{\left(t x_{i} x_{j}^{-1}, p\right)_{\infty}}
$$

where $(a, p)_{\infty}:=\prod_{j=0}^{\infty}\left(1-a p^{j}\right)$.

The Macdonald constant term identity (see [M1], p.20-21) says that the constant term of the Laurent series (8.5) (with respect to $x_{i}$ ) is given by

$$
\text { c.t. }\left(\Delta_{p, t}\right)=m ! \prod_{i<j} \frac{\left(t^{j-i} p, p\right)_{\infty}\left(t^{j-i}, p\right)_{\infty}}{\left(t^{j-i+1}, p\right)_{\infty}\left(t^{j-i-1} p, p\right)_{\infty}} .
$$


Setting in this identity $p=0$, we get

$$
\text { c.t. }\left(\Delta_{0, t}\right)=m ! \frac{(1-t)^{m}}{(1-t) \ldots\left(1-t^{m}\right)} .
$$

Substituting $t=q^{-2}$, we obtain the Lemma.

Define the expression

$$
I_{k, m}=q^{-k(\lambda+\mu+2 m)-\frac{k(k-1)}{2}} \frac{k !}{[k]_{q} !}, 0 \leq k \leq m,
$$

and the differential form

$$
\begin{gathered}
\Omega_{k, m}=\prod_{j=1}^{k} \frac{\left(T_{j} A^{-2} q^{\lambda+m}-q^{-\lambda-m}\right)\left(T_{j} A^{-2} q^{\mu+m}-q^{-\mu-m}\right)\left(1-T_{j} A^{-2} q^{2 m-2 k-2}\right)}{\left(1-T_{j} A^{2}\right)\left(1-T_{j} A^{-2} q^{2 m-2 k}\right)\left(1-T_{j} A^{-2} q^{-2}\right)} \times \\
(8.9) \quad \prod_{1 \leq i<j \leq k} \frac{\left(1-T_{i} T_{j}^{-1}\right)^{2}}{\left(1-T_{i} T_{j}^{-1} q^{-2}\right)\left(1-T_{i} T_{j}^{-1} q^{2}\right)} \wedge_{j=1}^{k} \frac{d T_{j}}{2 \pi \sqrt{-1} T_{j}} .
\end{gathered}
$$

Lemma 8.3. One has

$$
\int_{\left|T_{j}\right|=A^{2} q^{-2(m-k)}(1+\epsilon)} \Omega_{k, m}=
$$

$$
-k q^{-2(m-k)} \frac{\left(q^{\lambda+k}-q^{-\lambda-k}\right)\left(q^{\mu+k}-q^{-\mu-k}\right)\left(1-q^{-2}\right)}{\left(1-A^{4} q^{-2(m-k)}\right)\left(1-q^{-2(m-k+1)}\right)} \int_{\left|T_{j}\right|=A^{2} q^{-2(m-k+1)}(1+\epsilon)} \Omega_{k-1, m}+I_{k, m} .
$$

Proof. Let us perform the integration with respect to $T_{k}$, for fixed $T_{1}, \ldots, T_{k-1}$. It is obvious that the differential form $F_{k, m}$, as a function of $T_{k}$, has two simple poles inside the circle of integration $-T_{k}=A^{2} q^{2 k-2 m}$ and $T_{k}=0$. Therefore, the integral with respect to $T_{k}$ is equal to the sum of residues at these two poles. The residue at the first pole can be found by a direct computation and equals the first term on the right hand side of (8.10). The residue at zero equals to $I_{k, m}$ by Lemma 8.2. Lemma 8.3 is proved.

Now let us prove Theorem 8.1. Let us move the contour of integration in the definition of $I_{m}$ from $\left|T_{j}\right|=1$ to $\left|T_{j}\right|=A^{2}(1+\epsilon)$, via contours $\left|T_{j}\right|=B, A^{2}(1+\epsilon) \leq$ $B \leq 1$. On the way, we do not run into any poles, therefore, we have

$$
u_{m}(\lambda, \mu)=\left.q^{-\lambda \mu-m(m-1)} \int_{\left|T_{j}\right|=A^{2}(1+\epsilon)} \Omega_{m, m}\right|_{A=q^{m}}
$$

(in the sense of analytic continuation). So, to prove Theorem 8.1, it is enough to compute $\left.\int_{\left|T_{j}\right|=A^{2}(1+\epsilon)} \Omega_{m, m}\right|_{A=q^{m}}$. We do it by using the recursive relation given in Lemma 8.3. Namely, we have 
Lemma 8.4. One has

$$
\left.\int_{\left|T_{j}\right|=A^{2} q^{-2(m-k)}(1+\epsilon)} \Omega_{k, m}\right|_{A=q^{m}}=\sum_{j=0}^{k} c_{k j} I_{j, m},
$$

where

$$
c_{k j}=(-1)^{k-j}\left(1-q^{-2}\right)^{k-j} \frac{k !}{j !} \prod_{i=j+1}^{k} \frac{\left(q^{\lambda+i}-q^{-\lambda-i}\right)\left(q^{\mu+i}-q^{-\mu-i}\right)}{\left(1-q^{2(i+m)}\right)\left(1-q^{2(i-m-1)}\right)} q^{2(i-m)} .
$$

Proof. The proof is a straightforward induction in $k$ using Lemma 8.3.

Substituting $k=m$ in Lemma 8.4, and using the definition of $I_{k, m}$, we find the following expression for $u_{m}$ :

$$
u_{m}(\lambda, \mu)=q^{-m(3 m-1)} \frac{m !}{[2 m]_{q} !}\left(q-q^{-1}\right)^{-m} \times
$$

$q^{-\lambda \mu} \sum_{l=0}^{m} q^{-l(\lambda+\mu)-l(l-1) / 2}\left(q-q^{-1}\right)^{l} \frac{[m+l]_{q} !}{[l]_{q} ![m-l]_{q} !} \prod_{i=j+1}^{m}\left(q^{\lambda+i}-q^{-\lambda-i}\right)\left(q^{\mu+i}-q^{-\mu-i}\right)$.

Comparing (8.14) with (7.12), we get Theorem 8.1.

\section{Trace FUnCtions AND Macdonald theORY}

In this section, following [EK1] and [FV1], we will connect the results of this paper with the Macdonald-Ruijsenaars theory.

We restrict ourselves to the case of $\mathfrak{g}=s l_{n}, N=1$, and let $V$ be the q-analogue of the representation $S^{m n} \mathbb{C}^{n}$. The zero-weight subspace of this representation is 1-dimensional, so the function $\Psi_{V}$ can be regarded as a scalar function. We will denote this scalar function by $\Psi_{m}(q, \lambda, \mu)$

Recall the definition of Macdonald operators [M2,EK1]. They are operators on the space of functions $f\left(\lambda_{1}, \ldots, \lambda_{n}\right)$ which are invariant under simultaneous shifting of the variables, $\lambda_{i} \rightarrow \lambda_{i}+c$, and have the form

$$
M_{r}=\sum_{I \subset\{1, \ldots, n\}:|I|=r}\left(\prod_{i \in I, j \notin I} \frac{t q^{2 \lambda_{i}}-t^{-1} q^{2 \lambda_{j}}}{q^{2 \lambda_{i}}-q^{2 \lambda_{j}}}\right) T_{I},
$$

where $T_{I} \lambda_{j}=\lambda_{j}$ if $j \notin I$ and $T_{I} \lambda_{j}=\lambda_{j}+1$ if $j \in I$. Here $q, t$ are parameters. We will assume that $t=q^{m+1}$, where $m$ is a nonnegative integer.

It is known [M2] that the operators $M_{r}$ commute. From this it can be deduced that for a generic $\mu=\left(\mu_{1}, \ldots, \mu_{n}\right), \sum \mu_{i}=0$, there exists a unique power series $f_{m 0}(q, \lambda, \mu) \in \mathbb{C}\left[\left[q^{\lambda_{2}-\lambda_{1}}, \ldots, q^{\lambda_{n}-\lambda_{n-1}}\right]\right]$ such that the series $f_{m}(q, \lambda, \mu):=$ $q^{2(\lambda, \mu-m \rho)} f_{m 0}(q, \lambda, \mu)$ satisfies difference equations

$$
M_{r} f_{m}(q, \lambda, \mu)=\left(\sum_{I \subset\{1, \ldots, n\}:|I|=r} q^{2 \sum_{i \in I}(\mu+\rho)_{i}}\right) f_{m}(q, \lambda, \mu) .
$$

Remark. The series $f_{m 0}$ is convergent to an analytic (in fact, a trigonometric) function.

The following theorem is contained in [EK1]. 
Theorem 9.1. ([EK1], Theorem 5) One has

$$
f_{m}(q, \lambda, \mu)=\gamma_{m}(q, \lambda)^{-1} \Psi_{m}\left(q^{-1},-\lambda, \mu\right),
$$

where

$$
\gamma_{m}(q, \lambda):=\prod_{i=1}^{m} \prod_{l<j}\left(q^{\lambda_{l}-\lambda_{j}}-q^{2 i} q^{\lambda_{j}-\lambda_{l}}\right) .
$$

Remark. The exact statement of Theorem 5 of [EK1], in our conventions, is that the function $f_{m}(q, \lambda, \mu) \gamma_{m}(q, \lambda)$ is equal to $\left.\operatorname{Tr}\right|_{M_{\mu}}\left(\Phi_{\mu}^{V}\left(q^{-1}\right) q^{2 \lambda}\right)$, which is equivalent to Theorem 9.1.

Let $\mathcal{D}_{W}\left(q^{-1},-\lambda\right)$ denote the difference operator, obtained from the operator $\mathcal{D}_{W}$ defined in Section 1 by the transformation $q \rightarrow q^{-1}$ and the change of coordinates $\lambda \rightarrow-\lambda$. Let $\Lambda^{r} \mathbb{C}^{n}$ denote the q-analog of the $\mathrm{r}$-th fundamental representation of $s l_{n}$.

\section{Corollary 9.2.}

$$
\mathcal{D}_{\Lambda^{r} \mathbb{C}^{n}}\left(q^{-1},-\lambda\right)=\delta_{q}(\lambda) \gamma_{m}(q, \lambda) \circ M_{r} \circ \gamma_{m}(q, \lambda)^{-1} \delta_{q}(\lambda)^{-1}
$$

Proof. This follows from Theorem 9.1 and Theorem 1.1.

In conclusion of this section we would like to make several important remarks.

Remark 1. Corollary 9.2 is a degenerate (trigonometric) case of Theorem 5.2 in [FV1], which says that the elliptic Ruijsenaars operators are transfer matrices of the elliptic quantum $s l_{n}$ acting in $V[0]$. Thus, Theorem 1.1 and Theorem 9.1 immediately imply the trigonometric case of Theorem 5.2 of [FV1] (i.e. the case without spectral parameter).

Remark 2. Conversely, the trigonometric case of Theorem 5.2 of [FV1] together with Theorem 1.1 immediately implies Theorem 9.1 (and many other results of [EK1]). This is a "direct" proof of Theorem 9.1, in the sense that it involves (unlike the original proof of [EK1]) a direct computation of the radial parts of the central elements of $U_{q}(\mathfrak{g})$. (Another direct proof of Theorem 9.1 is given in [Mi], where the radial part of the central element corresponding to the vector representation is computed).

Remark 3. The line of argument discussed in Remark 2 can be extended to the elliptic case. Namely, combining an elliptic analogue of Theorem 1.1 (for affine Lie algebras at the critical level), and Theorem 5.2 of [FV1], one can prove an elliptic analogue of Theorem 9.1, which says that the radial parts of the central elements of $U_{q}\left(\widehat{s l_{n}}\right)$ at the critical level corresponding to evaluation modules $\Lambda^{r} \mathbb{C}^{n}(z)$, acting on functions with values in $V[0]$, are elliptic Ruisjsenaars operators. This has been a conjecture for a number of years (see e.g. [Mi], p.415). We plan to do this in a subsequent paper of this series.

Remark 4. In many arguments of this paper, Verma modules $M_{\mu}$ can be replaced with finite dimensional irreducible modules $L_{\mu}$ with sufficiently large highest weight, and one can prove analogs of Theorems 1.1-1.5 in this situation (in the same way). In particular, one may set $\hat{\Psi}_{m}(q, \lambda, \mu)=\operatorname{Tr}\left(\hat{\Phi}_{\mu}^{V} q^{2 \lambda}\right)$, where $\hat{\Phi}_{\mu}^{V}: L_{\mu} \rightarrow L_{\mu} \otimes V \otimes V^{*}[0]$ is the intertwiner with highest coefficient 1 (Such an 
operator exists iff $\mu-m \rho \geq 0$, see [EK1]). Then one can show analogously to Theorem 9.1 (see [EK1]) that the function $\hat{f}_{m}(q, \lambda, \mu):=\gamma_{m}(q, \lambda)^{-1} \hat{\Psi}_{m}\left(q^{-1},-\lambda, \mu+m \rho\right)$ is the Macdonald polynomial $P_{\mu}\left(q, t, q^{2 \lambda}\right)$ with highest weight $\mu$ ( $\mu$ is a dominant integral weight). In this case, Theorem 1.1 says that Macdonald's polynomials are eigenfunctions of Macdonald's operators, Theorem 1.2 gives recursive relations for Macdonald's polynomials with respect to the weight (for $s l(2)$ - the usual 3-term relation for orthogonal polynomials), and Theorem 1.3 is the Macdonald symmetry identity (see [M2]). (This representation theoretic derivation of the symmetry identity is somewhat different from the one in [EK2], where a pictorial argument is used.)

\section{Limiting CASES.}

In this section we will discuss various degenerations of the function $F_{V_{1}, \ldots, V_{N}}(q, \lambda, \mu)$, and the corresponding degenerate versions of Theorems 1.1-1.5. The main limiting cases we will be interested in are the classical limit, and the rational limit. The classical limit corresponds to passing from $U_{q}(\mathfrak{g})$ to $\mathfrak{g}$ in the trace construction; in this limit the function $F$ depends rationally on $\mu$ but trigonometrically of $\lambda$. This limit corresponds to the theory of spherical functions on the Lie group $G$ associated with $\mathfrak{g}$, which is discussed in [EFK1]. In the rational limit, which corresponds to the theory of spherical functions on $\mathfrak{g}$ rather than $G$, the function $F$ becomes rational in both $\lambda$ and $\mu$, restoring the symmetry. In this limit, the function $F$ is the Baker-Akhiezer function for a multivariable bispectral problem (see [Be]).

\subsection{The classical (KZB) limit.}

Let

$$
F_{V_{1}, \ldots, V_{N}}^{c}(\lambda, \mu)=\lim _{t \rightarrow 0} F_{V_{1}, \ldots, V_{N}}\left(q=e^{t}, \frac{\lambda}{2 t}, \mu\right) .
$$

We will call this limit the classical limit. The existence of this limit follows from Proposition 10.1 below.

Remark. Here and below we write the dependence of functions on $q$ explicitly, since in this section $q$ is allowed to vary.

Example 1. If $\mathfrak{g}=s l(2), \mathrm{N}=1$, and $V=V_{1}$ is the 3 -dimensional representation, we have

$$
F_{V}^{c}(\lambda, \mu)=e^{-\lambda \mu / 2} \frac{\mu}{\mu-1}\left(1-\frac{1}{\mu} \frac{1+e^{\lambda}}{1-e^{\lambda}}\right)
$$

The classical limit is obtained when in the situation of Section 1 we take the ordinary enveloping algebra $U(\mathfrak{g})$ instead of the quantized one $U_{q}(\mathfrak{g})$.

More precisely, let $\Phi_{\mu}^{V}$ be intertwining operators for $U(\mathfrak{g})$ defined as in Section 1 , and set

$$
\Psi_{V_{1}, \ldots, V_{N}}^{c}(\lambda, \mu)=\operatorname{Tr}\left(\left(\Phi_{\mu+\sum_{i=2}^{N} h^{(* i)}}^{V_{1}} \otimes 1^{N-1}\right) \ldots \Phi_{\mu}^{V_{N}} e^{\lambda}\right)
$$

Also, set

$$
\delta(\lambda)=e^{(\lambda, \rho)} \prod_{\alpha>0}\left(1-e^{-(\lambda, \alpha)}\right)
$$

and let $Q^{c}(\mu)$ be the limit of $\mathbb{Q}(\mu)$ as $q \rightarrow 1$ (i.e. it is defined as in Section 1 from representation theory of $U(\mathfrak{g}))$. Then we have 


\section{Proposition 10.1.}

(10.5)

$F_{V_{1}, \ldots, V_{N}}^{c}(\lambda, \mu)=\delta(\lambda)\left[\mathbb{Q}^{-1}(\mu)^{(* N)} \otimes \ldots \otimes \mathbb{Q}^{-1}\left(\mu-h^{(* 2 \ldots * N)}\right)^{(* 1)}\right] \Psi_{V_{1}, \ldots, V_{N}}^{c}(\lambda,-\mu-\rho)$.

Proof. The proof is straightforward.

Let us now look at the degenerations of the properties of $F_{V_{1}, \ldots, V_{N}}$ in the classical limit. We start with the analogue of Theorem 1.1.

First of all, we have the following analogue of Proposition 2.1, which is proved analogously to Proposition 2.1.

Proposition 10.2. (i) For any element $X$ of $U(\mathfrak{g})$ there exists a unique differential operator $D_{X}$ acting on $V[0]$-valued functions, such that

$$
\operatorname{Tr}\left(\Phi_{\mu}^{V} X e^{\lambda}\right)=D_{X} \operatorname{Tr}\left(\Phi_{\mu}^{V} e^{\lambda}\right) .
$$

(ii) If $X$ is central then $D_{X Y}=D_{Y} D_{X}$ for all $Y \in U(\mathfrak{g})$. In particular, if $X, Y$ are central then $D_{X} D_{Y}=D_{Y} D_{X}$.

The operator $D_{X}$ can be computed explicitly for any element $X$, but in general the answer is complicated. However, if $X$ is the quadratic Casimir $C$, the answer is easy to write down. Namely, define $\tilde{D}_{X}=\delta(\lambda) D_{X} \delta(\lambda)^{-1}$. Then we have $([\mathrm{E}],[\mathrm{ES} 1])$ :

$$
\tilde{D}_{C}=\Delta_{\mathfrak{h}}-\sum_{\alpha>0} \frac{f_{\alpha} e_{\alpha}}{2 \sinh ^{2} \frac{1}{2}(\lambda, \alpha)}-(\rho, \rho)
$$

where $f_{\alpha}, e_{\alpha}$ are root generators such that $\left(e_{\alpha}, f_{\alpha}\right)=1$, and $\Delta_{\mathfrak{h}}$ is the Laplacian on the Cartan subalgebra associated with the standard invariant form.

Thus, we have the following classical analogue of Theorem 1.1:

Theorem 10.3. For any $X$ in the center of $U(\mathfrak{g})$, let $p_{X}$ be the symmetric polynomial on $\mathfrak{h}^{*}$ such that $\left.X\right|_{M_{\mu}}=p_{X}(\mu+\rho)$. Then we have

$$
\tilde{D}_{X}^{\lambda} F_{V_{1}, \ldots, V_{N}}^{c}(\lambda, \mu)=p_{X}(-\mu) F_{V_{1}, \ldots, V_{N}}^{c}(\lambda, \mu) \text {. }
$$

In particular,

$$
\left(\Delta_{\mathfrak{h}}-\sum_{\alpha>0} \frac{f_{\alpha} e_{\alpha}}{2 \sinh ^{2} \frac{1}{2}(\lambda, \alpha)}\right) F_{V_{1}, \ldots, V_{N}}^{c}(\lambda, \mu)=(\mu, \mu) F_{V_{1} \ldots, V_{N}}^{c}(\lambda, \mu)
$$

Formula (10.9) was obtained in [E,ES1], but it can also be derived by taking the classical limit in Theorem 1.1.

Now let us consider the classical analogue of Theorem 1.2. Let $\mathcal{D}_{W}^{\vee, c}$ denote the difference operators defined by formula (1.12) for $q=1$ (i.e. $\mathbb{R}(\mu)$ are the exchange matrices for $U(\mathfrak{g})$ with $\mu$ replaced by $-\mu-\rho)$. Then we have the following result, obtained by passing to the limit in Theorem 1.2. 


\section{Theorem 10.4.}

$$
\mathcal{D}_{W}^{\vee, c, \mu} F_{V_{1} \ldots V_{N}}^{c}(\lambda, \mu)=\chi_{W}\left(e^{\lambda}\right) F_{V_{1} \ldots V_{N}}^{c}(\lambda, \mu),
$$

Example 2. In the case of Example 1, Theorems 10.3 and 10.4 have the form

$$
\left(\frac{\partial^{2}}{\partial \lambda^{2}}-\frac{1}{2 \sinh ^{2}(\lambda / 2)}\right) F_{V}^{c}(\lambda, \mu)=\frac{\mu^{2}}{4} F_{V}^{c}(\lambda, \mu)
$$

and

$$
\left(T+\frac{(\mu-2)(\mu+1)}{\mu(\mu-1)} T^{-1}\right) F_{V}^{c}(\lambda, \mu)=\left(e^{\lambda / 2}+e^{-\lambda / 2}\right) F_{V}^{c}(\lambda, \mu)
$$

(where $T$ is the shift by 1 in $\mu$ ), which is easily checked from (10.2).

Now consider the classical limit of Theorem 1.3. For this purpose introduce the classical dynamical r-matrix $r(\lambda)$, which is the classical limit of the exchange matrix $R(q, \lambda)$. This matrix is defined by the formula

$$
R\left(q=e^{t}, \frac{\lambda}{2 t}\right)=1-2 r(\lambda) t+O\left(t^{2}\right)
$$

and equals to

$$
r(\lambda)=-\frac{1}{2} \Omega+\frac{1}{2} \sum_{\alpha>0} \operatorname{cotanh} \frac{1}{2}(\lambda, \alpha) e_{\alpha} \wedge f_{\alpha},
$$

where $\Omega$ is the Casimir tensor (see $[\mathrm{EV}]$ ). Taking the quasiclassical limit in Theorem 1.3 , and using that $r(-\lambda)=r^{21}(\lambda)$, we obtain the following result.

Theorem 10.5. For any $j=1, \ldots, N$, one has

$$
\begin{gathered}
{\left[\frac{\partial}{\partial h^{(j)}}-\left(\sum_{l<j} r_{l j}(\lambda)-\sum_{l>j} r_{j l}(\lambda)\right)\right] F_{V_{1}, \ldots, V_{N}}^{c}(\lambda, \mu)=} \\
{\left[\left(\mu+\frac{1}{2} \sum x_{i}^{2}\right)_{* j}+\sum_{l<j} \sum x_{i}^{(* j)} \otimes x_{i}^{(* l)}\right] F_{V_{1}, \ldots, V_{N}}^{c}(\lambda, \mu),}
\end{gathered}
$$

where $\frac{\partial}{\partial h^{(j)}} X(\lambda)=\frac{\partial}{\partial \nu} X(\lambda)$ if $X$ is a tensor-valued function whose $j$-th component has weight $\nu$.

The last equation is the trigonometric limit of the KZB equation, which is why the classical limit is called "the KZB limit".

Let us now consider the classical limit of Theorem 1.4. Let $K_{j}^{\vee, c}$ be the difference operators defined by formula (1.17) for $q=1$ (i.e. $\mathbb{R}(\mu)$ are exchange matrices for $U(\mathfrak{g})$ with $\mu$ replaced by $-\mu-\rho)$. Then we have the following result, obtained by passing to the limit in Theorem 1.4.

Theorem 10.6. One has

$$
K_{j}^{\vee, c} F_{V_{1} \ldots V_{N}}^{c}(\lambda, \mu)=\left(e^{\lambda}\right)_{j} F_{V_{1} \ldots V_{N}}^{c}(\lambda, \mu) .
$$

Finally, Theorem 1.5 does not have an analogue in the classical limit. In this limit, the symmetry between $\lambda$ and $\mu$ is destroyed, since $F^{c}$ is a product of $e^{(\lambda, \mu)}$ with a function that is trigonometric in $\lambda$ but rational in $\mu$. 


\subsection{The rational limit.}

The rational limit is a further degeneration of the classical limit. Namely, let

$$
F_{V_{1}, \ldots, V_{N}}^{r}(\lambda, \mu)=\lim _{\gamma \rightarrow 0} F_{V_{1}, \ldots, V_{N}}^{c}(\lambda \gamma, \mu / \gamma)
$$

We will call this limit the rational limit. The existence of this limit and the fact that $\operatorname{det}\left(F^{r}\right) \neq 0$ can be deduced from Corollary 3.3 in [ES1].

Example 3. If $\mathfrak{g}=\operatorname{sl}(2), \mathrm{N}=1$, and $V=V_{1}$ is the 3 -dimensional representation, we have

$$
F_{V}^{r}(\lambda, \mu)=e^{-\lambda \mu / 2}\left(1+\frac{2}{\lambda \mu}\right)
$$

The degeneration of Theorem 1.1 in this limit is the following theorem. Let $\tilde{D}_{X}^{r}$ be the rational limit of $\tilde{D}_{X}$, i.e. $\tilde{D}_{X}^{r}(\lambda)$ is the leading coefficinet of $\tilde{D}_{X}(\gamma \lambda)$ as $\gamma \rightarrow 0$. For instance,

$$
\tilde{D}_{C}^{r}=\Delta_{\mathfrak{h}}-\sum_{\alpha>0} \frac{2 f_{\alpha} e_{\alpha}}{(\lambda, \alpha)^{2}}
$$

Theorem 10.7. For any $X$ in the center of $U(\mathfrak{g})$, let $p_{X}$ be the symmetric polynomial on $\mathfrak{h}^{*}$ such that $\left.X\right|_{M_{\mu}}=p_{X}(\mu+\rho)$. Let $p_{X}^{r}$ be the top degree component of $p_{X}$. Then we have

$$
\tilde{D}_{X}^{r, \lambda} F_{V_{1}, \ldots, V_{N}}^{r}(\lambda, \mu)=p_{X}^{r}(-\mu) F_{V_{1}, \ldots, V_{N}}^{r}(\lambda, \mu)
$$

In particular,

$$
\left(\Delta_{\mathfrak{h}}-\sum_{\alpha>0} \frac{2 f_{\alpha} e_{\alpha}}{(\lambda, \alpha)^{2}}\right) F_{V_{1}, \ldots, V_{N}}^{r}(\lambda, \mu)=(\mu, \mu) F_{V_{1} \ldots, V_{N}}^{r}(\lambda, \mu)
$$

The degeneration of Theorem 1.2 looks as follows:

Theorem 10.8. Equations (10.17),(10.18) are satisfied for the function $F_{V_{N}^{*}, \ldots, V_{1}^{*}}^{r, *}$.

Example 4. In the situation of Example 3, Theorems 10.7, 10.8 have the form

$$
\begin{aligned}
& \left(\frac{\partial^{2}}{\partial \lambda^{2}}-\frac{2}{\lambda^{2}}\right) F_{V}^{r}(\lambda, \mu)=\frac{\mu^{2}}{4} F_{V}^{r}(\lambda, \mu), \\
& \left(\frac{\partial^{2}}{\partial \mu^{2}}-\frac{2}{\mu^{2}}\right) F_{V}^{r}(\lambda, \mu)=\frac{\lambda^{2}}{4} F_{V}^{r}(\lambda, \mu),
\end{aligned}
$$

which is easily checked from (10.15).

Using the asymptotics of $F_{V_{1}, \ldots, V_{N}}^{r}(\lambda, \mu)$ at infinity, similarly to arguments of Section 5, one can deduce from Theorems 10.7,10.8 the following analog of Theorem 1.5 (the symmetry theorem): 
Theorem 10.9. The function $F_{V_{1} \ldots V_{N}}^{r}$ is symmetric:

$$
F_{V_{1} \ldots V_{N}}^{r}(\lambda, \mu)=F_{V_{N}^{*} \ldots V_{1}^{*}}^{r, *}(\mu, \lambda),
$$

Thus, the symmetry, lost in the first limit, is restored after taking the second limit.

Remark 1. Another proof of Theorem 10.8 is based on representation of the above sequence of two limits as a single limiting procedure, which is symmetric in $\lambda$ and $\mu$. Namely, one can show that

$$
F_{V_{1}, \ldots, V_{N}}^{r}(\lambda, \mu)=\lim _{s, t \rightarrow 0} F_{V_{1}, \ldots, V_{N}}\left(q=e^{s t / 2}, \frac{\lambda}{t}, \frac{\mu}{s}\right),
$$

after which Theorem 10.9 follows from Theorem 1.5.

Remark 2. Theorems 10.7, 10.8 show that the function $F_{V_{1}, \ldots, V_{N}}^{r}(\lambda, \mu)$ is a solution of the matrix bispectral problem in several variables (on the bispectral problem, see e.g. [DG,G]). The Baker-Akhiezer function of the rational Calogero system of type $A$, which is a known solution of the multidimensional bispectral problem ([VSC], see also [Be]), is a special case of $F_{V_{1}, \ldots, V_{N}}^{r}(\lambda, \mu)(N=1, V=$ $\left.V_{1}=S^{m n} \mathbb{C}^{n}\right)$.

Finally, let us consider the rational limit of Theorems 1.3, 1.4. To formulate the analog of Theorem 1.3, introduce the rational limit of the classical dynamical r-matrix, $r^{0}(\lambda)=\lim _{\gamma \rightarrow 0} \gamma r(\gamma \lambda)$. It has the form

$$
r^{0}(\lambda)=\sum_{\alpha>0} \frac{e_{\alpha} \wedge f_{\alpha}}{(\lambda, \alpha)}
$$

Taking the rational limit in Theorem 10.4, we get

Theorem 10.10. For any $j=1, \ldots, N$, one has

$$
\begin{gathered}
{\left[\frac{\partial}{\partial h^{(j)}}-\left(\sum_{l<j} r_{l j}^{0}(\lambda)-\sum_{l>j} r_{j l}^{0}(\lambda)\right)\right] F_{V_{1}, \ldots, V_{N}}^{r}(\lambda, \mu)=} \\
\mu_{* j} F_{V_{1}, \ldots, V_{N}}^{r}(\lambda, \mu) .
\end{gathered}
$$

The analogue of Theorem 1.4 is

Theorem 10.11. Equation (10.22) is satisfied for the function $F_{V_{N}^{*}, \ldots, V_{1}^{*}}^{r * *}$.

\subsection{The $q K Z$ and $K Z$ limits.}

Assume that $|q|<1$. The $\mathrm{qKZ}$ limit is defined by

$$
F_{V_{1}, \ldots, V_{N}}^{q K Z}(\lambda, \mu)=\lim _{\left(\lambda, \alpha_{i}\right) \rightarrow-\infty} q^{2(\lambda, \mu)} F_{V_{1}, \ldots, V_{N}}(\lambda, \mu) .
$$

It is easy to check using Theorem 50 of $[\mathrm{EV}]$ that

$$
\begin{gathered}
F_{V_{1}, \ldots, V_{N}}^{q K Z}(\lambda, \mu)= \\
{\left[\mathbb{Q}^{-1}(\mu)^{(* N)} \otimes \ldots \otimes \mathbb{Q}^{-1}\left(\mu-h^{(* 2 \ldots * N)}\right)^{(* 1)}\right]\left\langle\left(\Phi_{\mu+\sum_{i=2}^{N} h_{1}(* i)}^{V_{1}} \otimes 1^{N-1}\right) \ldots \Phi_{\mu}^{V_{N}}\right\rangle=} \\
(10.24) \\
{\left[\mathbb{Q}^{-1}(\mu)^{(* N)} \otimes \ldots \otimes \mathbb{Q}^{-1}\left(\mu-h^{(* 2 \ldots * N)}\right)^{(* 1)}\right] \mathbb{J}^{1 \ldots N}(\mu)^{*},} \\
34
\end{gathered}
$$


where $\langle$,$\rangle denotes the highest matrix element. (The last expression is an endomor-$ phism of $\left(V_{N}^{*} \otimes \ldots \otimes V_{1}^{*}\right)[0]$, which is regarded as an element of $\left.\left(V_{1} \otimes \ldots \otimes V_{N}\right)[0] \otimes\left(V_{N}^{*} \otimes \ldots \otimes V_{1}^{*}\right)[0]\right)$. In particular, this function is independent on $\lambda$.

Let us now consider the behavior of the equations given by Theorems 1.1-1.5 in the qKZ limit.

The MR equations given by Theorem 1.1 become trivial. Namely, when $\left(\lambda, \alpha_{i}\right) \rightarrow$ $-\infty$, one has $\mathbb{J}(\lambda) \rightarrow 1$, and hence $\mathbb{R}(\lambda) \rightarrow \mathcal{R}^{21}$. The matrix $\mathcal{R}^{21}$ is triangular, so only its diagonal part contributes to the trace. Inspection of this diagonal part shows that $\lim _{\left(\lambda, \alpha_{i}\right) \rightarrow-\infty} \mathcal{D}_{W}=\sum_{\nu} \operatorname{dim} W[\nu] T_{\nu}$, and the limiting equation is

$$
\left(\sum_{\nu} \operatorname{dim} W[\nu] q^{-2(\nu, \mu)} T_{\nu}\right) F^{q K Z}=\chi_{W}\left(q^{-2 \mu}\right) F^{q K Z}
$$

(where $T_{\nu}$ is the shift of $\lambda$ ), which is a trivial consequence of the fact that $F^{q K Z}$ is independent on $\lambda$.

The dual MR equations given by Theorem 1.2 have a slightly more interesting limit. It is easy to see that the only term on each side of (1.11) which survives in the limit is the term corresponding to the lowest weight $\nu_{W}$ of $W$. Therefore, the limiting equation has the form

$\left(v_{W}^{*} \otimes 1, \mathbb{R}_{W V_{N}^{*}}^{01}\left(\mu+h^{(* 1 \ldots * N-1)}\right) \ldots \mathbb{R}_{W V_{1}^{*}}^{0 N}(\mu)\left(v_{W} \otimes 1\right)\right) F_{V_{1}, \ldots, V_{N}}^{q K Z}\left(\mu+\nu_{W}\right)=F_{V_{1}, \ldots, V_{N}}^{q K Z}(\mu)$.

The qKZB equations become the trigonometric limit of the $\mathrm{qKZ}$ equations. Namely, for all $j=1, \ldots, N$ we have

$$
\left[\mathcal{R}_{j, j+1}^{-1} \ldots \mathcal{R}_{j N}^{-1}\left(q^{-2 \mu}\right)_{j} \mathcal{R}_{1 j} \ldots \mathcal{R}_{j-1, j} \otimes D_{j}\right] F_{V_{1}, \ldots, V_{N}}^{q K Z}(\mu)=F_{V_{1}, \ldots, V_{N}}^{q K Z}(\mu) .
$$

If $N=2$, these equations are closely related to the Arnaudon-Buffenoir-RagoucyRoche (ABRR) equation (see Lemma 2.4). In general, they are essentially the $\mathrm{N}$-component version of the ABRR equation.

Remark. The dual qKZB equations do not seem to have a reasonable qKZ limit. Also, the symmetry relation (Theorem 1.5) does not hold in the qKZ limit since the function depends on $\mu$ and not on $\lambda$.

The KZ limit is obtained from the qKZ limit as $q \rightarrow 1$, in which case the $\mathrm{qKZ}$ equations degenerate into the trigonometric $\mathrm{KZ}$ equations (the quasiclassical limit of the ABRR equation). We leave it to the reader to derive the limiting equations in this case.

We plan to consider these limits in more detail in another paper in the more interesting case of affine Lie algebras and quantum affine algebras.

\section{REFERENCES}

[ABRR] D.Arnaudon, E.Buffenoir, E.Ragoucy, and Ph.Roche, Universal Solutions of quantum dynamical Yang-Baxter equations, q-alg/9712037.

[Be] Berest Yu., Huygens' principle and the bispectral problem, CRM Proc. Lect. Notes 14 (1998), 11-30.

[Ber] Bernard, D., On the Wess-Zumino-Witten models on the torus, Nucl. Phys. B303 (1988), 77-93.

[C] Cherednik, I., Macdonald's evaluations conjectures and difference Fourier transform, Invent. Math 122 (1995), 119-145. 
[D] Drinfeld, V.G., On almost cocommutative Hopf algebras, Leningrad Math.J. 1 (1990), no. 2, $321-342$.

[DG] Duisetrmaat J.J., Grünbaum, F.A., Differential equations in the spectral parameter, Comm. Math. Phys. 103 (1986), 177-240.

[E] Etingof, P.I., Quantum integrable systems and representations of Lie algebras, hep-th 9311132, submitted to Journal of Mathematical Physics (1993).

[EFK] P. Etingof, I. Frenkel, and A. Kirillov Jr., Lectures on representation theory and KnizhnikZamolodchikov equations, AMS, 1998.

[EFK1] P. Etingof, I. Frenkel, and A. Kirillov Jr., Spherical functions on affine Lie groups, DMJ 10 (1995), no. 1, 59-90.

[EK1] Etingof, P.I. and Kirillov, A.A., Jr, Macdonald's polynomials and representations of quantum groups, Math. Res. Let. 1(3) (1994), 279-296.

[EK2] Etingof, P.I. and Kirillov, A.A., Jr, Representation-theoretic proof of the inner product and symmetry identities for Macdonald's polynomials, Comp.Math. 102 (1996), 179-202.

[EK3] Etingof, P.I. and Kirillov, A.A., Jr, On Cherednik-Macdonald-Mehta identities, math.QA 9712051, Electr. Res. Ann. (1997).

[EK4] Etingof, P.I. and Kirillov, A.A., Jr, On an affine analogue of of Jack and Macdonald polynomials, Duke Math.J. 78 (1995), no. 2.

[ESch] P.Etingof and O.Schiffmann,, Lectures on the dynamical Yang-Baxter equations, math.QA 9908064 (1999).

[ES] P.Etingof and K.Styrkas, Alqebraic integrability of Macdonald operators and representations of quantum groups, q-alg 9603022. Compos. Math 114 (1998), 125-152.

[ES1] P.Etingof, K.Styrkas, Algebraic integrability of Schrödinger operators and representations of Lie algebras, Compositio Mathematica 98 (1995), 91-112.

[EV] P.Etingof and A.Varchenko, Exchange dynamical quantum groups, q-alg 9801135, to appear in CMP (1998).

[F] G. Felder, Conformal field theory and integrable systems associated to elliptic curves, Proceedings of the International Congress of Mathematicians. Zürich 1994, p. 1247-1255, Birkhäuser, 1994; Elliptic quantum groups, preprint hep-th/9412207, to appear in the Proceedings of the ICMP, Paris 1994.

[FR] I. Frenkel and N. Reshetikhin, Quantum affine algebras and holonomic difference equations, Commun. Math. Phys. 146 (1992), 1-60.

[FTV1] G.Felder, V.Tarasov, A.Varchenko, Solutions of the elliptic QKZB equations and Bethe ansatz I, q-alg/9606005, in: Topics in Singularity Theory, V.I.Arnold's 60th Anniversary Collection, Advances in the Mathematical Sciences -34, AMS Translations, Series 2, 180 (1997), 45-76.

[FTV2] G.Felder, V.Tarasov, A.Varchenko, Monodromy of solutions of the elliptic quantum KnizhnikZamolodchikov-Bernard difference equations, , q-alg/9705017 (1997), 1-26.

[FV1] G. Felder and A. Varchenko, Elliptic quantum groups and Ruijsenaars models, q-alg 9704005 (1997).

[FV2] G. Felder and A. Varchenko, Quantum KZB heat equation, modular transformations, and $G L(3, Z), I$, math.QA/9809139 (1998).

[FV3] G. Felder and A. Varchenko, Resonance relations for solutions of the elliptic qKZB equations, fusion rules, and eigenvectors of transfer matrices of restricted interaction-round-a-face models, math.QA/9901111 (1999).

[FV4] G. Felder and_A. Varchenko, Quantum KZB heat equation, modular transformations, and $G L(3, Z)$, II, math.QA 9907061 (1999).

[FV5] G. Felder and A. Varchenko, The elliptic gamma-function, and $S L_{3}(\mathbb{Z}) \ltimes \mathbb{Z}^{3}$, to appear in math.QA (1999).

[G] F.A.Grünbaum, Some bispectral musings, CRM Proc. Lect. Notes 14 (1998), 31-45.

[JKOS] M.Jimbo, H.Konno, S.Odake, J.Shiraishi, Quasi-Hopf twistors for elliptic quantum groups, q-alg 9712029 (1997).

[K] A.Kirillov Jr., Traces of intertwining operators and Macdonald polynomials, PhD thesis, a-alg 9503012 (1995).

[K1] Kirillov, A.A. Jr, On an inner product in modular tensor categories, JAMS 9 (1996), no. 4.

[K2] Kirillov, A.A. Jr, On an inner product in modular tensor categories, II, Adv.Theor.Math.Phys 2 (1998).

[M1] I.Macdonald, Symmetric functions and orthogonal polynomials, AMS, Providence, 1998. 
[M2] Macdonald, I.G., A new class of symmetric functions, Publ. I.R.M.A. Strasbourg, 372/S-20, Actes 20 Séminaire Lotharingien (1988), 131-171.

[Mi] K.Mimachi, Macdonald's operator from the center of the quantized enveloping algebra $U_{q}(g l(N))$, IMRN 10 (1994), 415-424.

$[\mathrm{MV}]$ E. Mukhin and A. Varchenko, Solutions of the qKZB equations in tensor products of finite dimensional modules over the elliptic quantum group $E_{\tau, \eta} s l_{2}, 9712056$ (1997).

[R] Reshetikhin, N.Yu., Quasitriangle Hopf algebras and invariants of tangles, Leningrad Math J. 1 (1990), no. 2, 491-513.

[TV1] V. Tarasov and A. Varchenko, Geometry of q-Hypergeometric functions as a bridge between Yangians and Quantum Affine Algebras, Inv.Math. 128 (1997), 501-588.

[TV2] V. Tarasov and A. Varchenko, Geometry of q-Hypergeometric Functions, Quantum Affine Algebras and Elliptic Quantum Groups, q-alg/9703044, Asterisque 246 (1997), 1-135.

[VSC] Veselov, A.P., Styrkas, K.A., and Chalykh, O.A., Algebraic integrability for the Schrödinger equation and finite reflection groups, Theor. and Math. Physics 94 (1993), no. 2. 